\title{
Obfuscating Conjunctions
}

\author{
Zvika Brakerski* Guy N. Rothblum ${ }^{\dagger}$
}

\begin{abstract}
We show how to securely obfuscate the class of conjunction functions (functions like $\left.f\left(x_{1}, \ldots, x_{n}\right)=x_{1} \wedge \neg x_{4} \wedge \neg x_{6} \wedge \cdots \wedge x_{n-2}\right)$. Given any function in the class, we produce an obfuscated program which preserves the input-output functionality of the given function, but reveals nothing else.

Our construction is based on multilinear maps, and can be instantiated using the recent candidates proposed by Garg, Gentry and Halevi (EUROCRYPT 2013) and by Coron, Lepoint and Tibouchi (CRYPTO 2013). We show that the construction is secure when the conjunction is drawn from a distribution, under mild assumptions on the distribution. Security follows from multilinear entropic variants of the Diffie-Hellman assumption. We conjecture that our construction is secure for any conjunction, regardless of the distribution from which it is drawn. We offer supporting evidence for this conjecture, proving that our obfuscator is secure for any conjunction against generic adversaries.
\end{abstract}

*Stanford University, zvika@stanford.edu. Supported by a Simons Postdoctoral Fellowship and by DARPA.

${ }^{\dagger}$ Microsoft Research Silicon Valley, rothblum@alum.mit.edu. 


\section{Introduction}

Code obfuscation is the problem of compiling a computer program so as to make it unintelligible to an adversary, or impossible to reverse-engineer, while preserving its input-output functionality. Obfuscation has been of long-standing interest to both the cryptography and security communities. However, despite the importance of the problem, and its many exciting applications, very few techniques or effective heuristics are known. In particular, the theoretical study of the problem (in the "virtual black-box model" $\left.\left[\mathrm{BGI}^{+} 12\right]\right)$ led to a handful of known constructions, which apply to very limited classes of functions. These include the class of point functions, and extensions such as multi-point functions, "lockers" and constant-dimension hyperplanes.

In this work, we present an obfuscator for a new and different class: conjunction functions. These are functions that take $n$-bit strings as input and only accept if a subset of these bits are set to predefined values. Our construction relies on (asymmetric) multilinear maps, and is instantiated using the new candidate construction due to Garg, Gentry and Halevi [GGH13].

Previous Results. The goal of an obfuscator is generating a program that preserves the functionality of the original program, but reveals nothing else. One commonly used formalization of this objective is "virtual black box" obfuscation, due to Barak et al. [BGI $\left.{ }^{+} 12\right]$. Our work uses this formalization, as well as alternative formalizations from subsequent works (see below).

In their work, $\left[\mathrm{BGI}^{+} 12\right]$ also proved the impossibility of general-purpose obfuscators (i.e. ones that work for any functionality) in the virtual black box model. This impossibility result was extended in [GK05]. While these negative results show serious limitations on the possibility of general-purpose obfuscation, they focus on specific (often cryptographic or contrived) functionalities. Thus, they do not rule out that obfuscation may be possible for many programs of interest.

Positive results on obfuscation focus on specific, simple programs. One program family, which has received extensive attention, is that of "point functions": password checking programs that only accept a single input string, and reject all others. Starting with the work of Canetti [Can97], several works have shown obfuscators for this family under various assumptions [CMR98, LPS04, Wee05], as well as extensions [CD08, BC10]. Canetti, Rothblum and Varia [CRV10] showed how to obfuscate a function that checks membership in a hyperplane of constant dimension (over a large finite field). Other works showed how to obfuscate cryptographic function classes under different definitions and formalizations. These function classes include checking proximity to a hidden point [DS05a], vote mixing [AW07], and re-encryption [HRSV11]. Several works [Can97, CMR98, HMLS10, HRSV11] relaxed the security requirement so that obfuscation only holds for a random choice of a program from the family, we will also use this relaxation for one of our results. A different relaxation, known as "best-possible obfuscation", which allows the obfuscation to leak non black-box information was presented in [GR07].

This Work: Obfuscating Conjunctions. Our main contribution is a new obfuscator for conjunctions. A conjunction $C=(W, V)$ is a function on $n$ bit inputs, specified by a set $W \subseteq[n]$ of "wildcard" entries, and a vector $V \in\{0,1\}{ }^{n}$ of target values for non-wildcard entries. The conjunction accepts an input $\vec{x} \in\{0,1\}^{n}$ if for all $i \in([n] \backslash W), \vec{x}[i]=V[i]$, i.e. if for all non-wildcard entries in $\vec{x}$, their values equal those specified in $V$. We use the convention that if $W[i]=1$ then $V[i]=0$ (wildcard entries are ignored, so this does not effect the conjunction's functionality).

The class of conjunctions, while obviously quite limited, has a rich combinatorial and com- 
putational expressive power. They are studied in a multitude of settings throughout computer science (e.g. in learning theory [KV94]). One significant distinction from previous function classes for which obfuscators were known, is that a conjunction may ignore some of its input bits (the wildcard entries). An obfuscator for conjunctions needs to produce a program that hides which bits are ignored, and which ones are influential.

As an example of the applications of a conjunction obfuscator, consider the following setting. There are $k$ passwords, each controlling access to a particular type of resource. Each individual knows some subset of the $k$ passwords, which corresponds to the resources it is allowed to access. A gatekeeper wishes to check whether an individual has access to some combination of resources, i.e. whether the individual knows a particular subset $S \subset[k]$ of the passwords, without revealing to an observer which combination it is checking. A conjunction, which takes as input $k$ concatenated passwords, can check whether the passwords for resources in $S$ are correct, while ignoring passwords for resources not in $S$. An obfuscation of this conjunction can be made public, and used to check whether an individual has access to that combination of resources, without revealing which resources are being checked (nor, of course, what any of the passwords are).

\subsection{Our Construction and its Security}

The main tool in our construction is multilinear maps. In particular, we utilize a recent candidate for graded encoding (a generalization of multilinear maps) due to [GGH13]. ${ }^{1}$ We prove the security of our obfuscator when the conjunction is chosen from a distribution with sufficient entropy: namely, when sampling $C=(W, V)$ from the distribution, even given the wildcard locations $W$, there is sufficient (superlogarithmic) entropy in $V$. We stress that this does not imply that the attacker is allowed to learn $W$; on the contrary, we prove that if $C$ is drawn from a distribution with the aforementioned property, the adversary cannot learn anything, wildcard locations included. ${ }^{2}$ As noted above, here we follow several works [Can97, CMR98, HMLS10, HRSV11] which relax the security requirement to hold only when the circuit to be obfuscated is drawn from a distribution from a certain class (usually one with sufficient entropy).

We prove the above under two security assumptions on graded encodings schemes: The first is a translation of the SXDH assumption on bilinear groups to the setting of graded encoding schemes. ${ }^{3}$ The second assumption is reminiscent of "Canetti's Assumption" [Can97] on Diffie-Hellman groups, which was introduced for the purpose of obfuscating point functions.

We conjecture that the construction is secure for every conjunction, but we were unable to produce a proof based on a well-established assumption (naturally, one can always take the security of the obfuscator as an assumption). As supportive evidence for the conjectured security, we prove that the obfuscator is secure against generic adversaries: Ones that only use the group structure and not the representation of the group elements. This is similar to the generic group model of [Sho97, Mau05]. The proof of security against generic adversaries is non-trivial, and we view this as one of our main technical contributions. We note that previous works on obfuscation

\footnotetext{
${ }^{1}$ We use the asymmetric variant of the encoding scheme, where there are several distinct "source groups".

${ }^{2}$ We remark that in this case nothing at all can be learned from black-box access to the function since it is infeasible to find an accepting input. We also remark that, for example, the conjunctions used for the $k$-resource application above naturally satisfy this condition, because of the entropy in each password.

${ }^{3}$ This assumption is actually known to be false for the construction and formulation of [GGH13]. However, we show a more careful definition of the scheme and the assumption for which no attack is known. Also, no attack is known for the recent construction of Coron, Lepoint and Tibouchi [CLT13].
} 
[LPS04, CRV10] have also used the random oracle and generic group models to provide evidence for the security of constructions.

We proceed with an overview of our construction and results. As we explained, the obfuscator uses the recent construction of multi-linear maps via graded encoding schemes [GGH13]. We begin with a high-level overview on the properties of multilinear maps that will be used. We then proceed with an overview of our construction, and state our two main results.

Multilinear Maps and Graded Encoding Schemes: Background. We begin by recalling the notion of multilinear maps, due to Boneh and Silverberg [BS02]. Rothblum [Rot13] considered the asymmetric case, where the groups may be different (this is crucial for our construction).

Definition 1.1 (Asymmetric Multilinear Map [BS02, Rot13]). For $\tau+1$ cyclic groups $G_{1}, \ldots, G_{\tau}$, $G_{T}$ of the same order $p$, a $\tau$-multilinear map $e: G_{1} \times \ldots \times G_{\tau} \rightarrow G_{T}$ has the following properties:

1. For elements $\left\{g_{i} \in G_{i}\right\}_{i=1, \ldots, \tau}$, index $i \in[\tau]$ and integer $\alpha \in \mathbb{Z}_{p}$, it holds that:

$$
e\left(g_{1}, \ldots, \alpha \cdot g_{i}, \ldots, g_{\tau}\right)=\alpha \cdot g\left(g_{1}, \ldots, g_{\tau}\right)
$$

2. The map $e$ is non-degenerate: when its inputs are all generators of their respective groups $\left\{G_{i}\right\}$, then its output is a generator of the target group $G_{T}$.

Recently, [GGH13] suggested a candidate for graded encoding, a generalization of (symmetric or asymmetric) multilinear maps. See Section 2.2 for a more complete overview of these objects. For this introduction, we treat them as a generalization of asymmetric multilinear maps in the following way. For a $\tau$-multilinear map $e$, for the group $G_{i}$ of prime order $p$, we consider the ring $\mathbb{Z}_{p}$. For an element $\sigma \in \mathbb{Z}_{p}$, we can think of $g_{i}^{\sigma}$ as an "encoding" of $\sigma$ in $G_{i}$. We denote this by enc $i(\sigma)$. We note that this encoding is easy to compute, but (presumably) hard to invert. The multilinear map $e$ lets us take $\tau$ encodings $\left\{\text { enc }_{i}\left(\sigma_{i}\right)\right\}_{i \in[\tau], \sigma_{i} \in \mathbb{Z}_{p}}$, and compute the target group encoding enc ${ }_{T}\left(\prod_{i} \sigma_{i}\right)$. Graded encoding schemes afford a similar functionality, albeit with randomized and noisy encodings, and with a procedure for testing equality of encoded elements in the target group.

Our Construction. For a conjunction $C=(W, V)$ on $n$-bits inputs, the obfuscator uses the graded encoding scheme to obtain the above generalization to an $(n+1)$-multilinear map. For each input entry $i \in[n]$, the obfuscator picks ring elements $\left(\rho_{i, 0}, \rho_{i, 1}, \alpha_{i, 0}, \alpha_{i, 1}\right)$ distributed as follows: if $i \notin W$, namely the entry isn't a wildcard, then the ring elements are independent and uniformly random. If $i \in W$, namely the entry is a wildcard, then the ring elements are uniformly random under the constraint that $\alpha_{i, 0}=\alpha_{i, 1}$. After picking the ring elements, the obfuscator outputs two pairs of encodings for each $i \in[n]$ :

$$
\left\{\left(w_{i, b}=\operatorname{enc}_{i}\left(\rho_{i, b}\right), u_{i, b}=\operatorname{enc}_{i}\left(\rho_{i, b} \cdot \alpha_{i, b}\right)\right)\right\}_{i \in[n], b \in\{0,1\}}
$$

Note that if $i \in W$, then the ratio between the ring elements encoded in $u_{i, 0}$ and $w_{i, 0}$, is equal to the ratio between the ring elements encoded in $u_{i, 1}$ and $w_{i, 1}$ (these ratios are, respectively, $\alpha_{i, 0}$ and $\alpha_{i, 1}$, which are equal when $\left.i \in W\right)$. We remark that this part of the obfuscation depends only on the wildcards $W$, but not on the values $V$. 
To complete the obfuscation, the obfuscator picks independent and uniformly random ring element $\rho_{n+1}$, and outputs a pair of encodings:

$$
\left(w_{n+1}=\operatorname{enc}_{n+1}\left(\rho_{n+1}\right), u_{n+1}=\operatorname{enc}_{n+1}(\rho_{n+1} \cdot \underbrace{\prod_{i \in[n]} \alpha_{i, V[i]}}_{=\alpha_{n+1}})\right)
$$

To evaluate the obfuscated program on an input $\vec{x} \in\{0,1\}^{n}$, we test equality between two multilinear products: ${ }^{4}$

$$
e\left(\ldots, u_{i, \vec{x}[i]}, \ldots, w_{n+1}\right) \stackrel{?}{=} e\left(\ldots, w_{i, \vec{x}[i]}, \ldots, u_{n+1}\right)
$$

The full construction is in Section 3.

Correctness. Examining the two multilinear products in Eq. (1), the element encoded in the lefthand side is $\left(\prod_{i \in[n]} \rho_{i, \vec{x}[i]} \cdot \alpha_{i, \vec{x}[i]}\right) \cdot \rho_{n+1}$. The element encoded in the right-hand side is $\left(\prod_{i \in[n]} \rho_{i, \vec{x}[i]}\right.$. $\left.\alpha_{i, V[i]}\right) \cdot \rho_{n+1}$. Thus, Eq. (1) holds if and only if:

$$
\prod_{i \in[n]} \alpha_{i, \vec{x}[i]}=\prod_{i \in[n]} \alpha_{i, V[i]}
$$

For $i \in W$ we have $\alpha_{i, 0}=\alpha_{i, 1}$, the contributions from the $i$-th group to both products in Eq. (2) are identical. For $i \notin W$, the contribution from the $i$-th group in the left-hand side of Eq. (2) is $\alpha_{i, \vec{x}[i]}$. In the right-hand side, the contribution is $\alpha_{i, V[i]}$. Except for a negligible probability of error, Eq. (2) holds if and only if all these contributions are identical, i.e. if and only if $\forall i \notin W: \vec{x}[i]=V[i]$.

Security. Security is not as straightforward. A slightly misleading intuition for security, is that if a DDH-like assumption holds within each group $G_{i}$ separately, then no observer can distinguish from that group's encodings whether $\alpha_{i, 0}=\alpha_{i, 1}$. This is true for each group in isolation, but it is insufficient because the obfuscation also includes encodings, in group $G_{n+1}$, of items that are correlated with the items encoded in group $i$. The multilinear map $e$ might allow an adversary to distinguish whether the $i$-th entry is a wildcard.

For example, if in $C$ all the entries are wildcards, the adversary can pick a random input, run the obfuscation, see that it accepts, and then by flipping the input bits one-by-one it can determine that all of the entries are wildcards. This attack clearly demonstrates that (for some conjunctions) an adversary can determine which entries are wildcards and which aren't. Note, however, that (for the specific example of a conjunction that is all-wildcards) this could also be accomplished using black-box access to the conjunction.

Indeed, we prove the security of the obfuscator when the conjunction is drawn from a distribution, under mild assumptions on the distribution's entropy. We conjecture that the obfuscator is actually secure for any conjunction, and as supporting evidence we show that it is secure against generic adversaries. An overview on both results follows.

\footnotetext{
${ }^{4}$ For the candidate of [GGH13], the encodings are randomized, but there is a procedure for testing equality between encoded elements in the target group.
} 
Security for High Entropy. We prove the security of our scheme in the case where $C=(W, V)$ is drawn from a distribution where the entropy of $V$ given $W$ is superlogarithmic. We do so by resurrecting the flawed argument described above: We use the entropy to remove the dependence between the elements in $G_{n+1}$ and those in the other groups, and then apply DDH in each group.

We start by noting that this dependence is due to the relation

$$
\alpha_{n+1}=\prod_{i \in[n]} \alpha_{i, V[i]},
$$

and if we could replace $\alpha_{n+1}$ with a completely uniform variable, independent of the other $\alpha$ 's, we'd be done. To this end, we notice that Eq. (3) describes an (almost) pairwise independent hash function, whose seed are the values $\alpha_{i, b}$ and whose input is $V$. We show that such a hash function is a good entropy condenser, so that almost all of the entropy in $V$ is preserved in $\alpha_{n+1}$. (It is important to notice that the distinguisher has side information which depends on $W$, and therefore we must require that the conditional entropy is high.)

Once we establish that $\alpha_{n+1}$ has superlogarithmic entropy, we use a "Canetti-like Assumption" [Can97]: we assume a high-entropy element in the exponent of a random group generator is indistinguishable from uniform. ${ }^{5}$ We thus isolate $\alpha_{n+1}$ from the dependence on the other $\alpha$ 's, which allows us to apply DDH in groups $G_{1}, \ldots, G_{n}$, and obtain the final result: that the obfuscated program comes from a distribution that can be efficiently simulated.

The security proof is in Section 4.

Security in The Generic Model. We prove security against generic adversaries. A generic adversary is one that succeeds regardless of the representation of the encoding scheme. This is modeled by allowing it to only manipulate encodings in the graded encoding scheme via oracle access to an oracle for the operations that are available using the evparams parameters. We show that for any generic adversary $\mathcal{A}$, which takes as input an obfuscation and outputs a single bit, there exists a generic simulator $\mathcal{S}$ s.t. for any conjunction $C$, the adversary's output on an obfuscation of $C$ is statistically close to the simulator's output given only black-box access to $C$. The distribution of the adversary is taken over the choice of a random graded encoding scheme oracle: an oracle that represents each encoding in each group using a (long enough) uniformly random string.

In this model, since each element's encoding is uniformly random, and the obfuscation contains the encodings of distinct ring elements, the obfuscation of any conjunction is simply a collection of uniformly random strings. Thus simulating the obfuscator's output is easy. The main challenge is that the outputs to oracle calls on the string in the obfuscation are highly dependant on the conjunction $C$. It is thus not clear how the simulator can simulate the oracle's outputs. For example, each accepting input $\vec{x}$ for $C$ specifies two possible inputs to the oracle implementing the multilinear map, which should both yield the same encoding in the target group. Indeed, simulating the oracle call outputs proves challenging. Moreover, the more generalized notion of graded encoding schemes permits more general generic operations.

The simulator $\mathcal{S}$ operates as follows. It feeds the adversary $\mathcal{A}$ with a "dummy obfuscation" containing uniformly random strings. It then follows $\mathcal{A}$ 's calls to the graded encoding scheme (GES) oracle, and tries to simulate the output. For each call made by $\mathcal{A}$, we show how $\mathcal{S}$ can (efficiently) identify a polynomial size set $X$ of inputs, such that if $\forall \vec{x} \in X, C(\vec{x})=0$, then the oracle's output

\footnotetext{
${ }^{5}$ Wee [Wee05] showed that these types of assumptions (hardness given only super-logarithmic entropy) are essential even for obfuscating point functions.
} 
is essentially independent of $C$ and can be simulated. On the other hand, if there exists $\vec{x} \in X$ s.t. $C(\vec{x})=1$, then the simulator can use its black-box access to $C$ to identify this input,. Once an accepting input is identified, the simulator can further use its block-box access to $C$ to retrieve the conjunction's explicit description $(W, V)$ (see Claim 3.3 below). Once the simulator knows $(W, V)$ it can (perfectly) simulate the adversary's behavior. We view this proof of security for generic adversaries as one of our main technical contributions.

The full specification and treatment of the generic GES model, as well as the proof of security for generic adversaries, are in Section 5.

\section{Preliminaries}

Notation. We use $\Delta(\cdot, \cdot)$ to indicate total variation distance (statistical distance). We use $\overrightarrow{1}$ (respectively $\overrightarrow{0}$ ) to denote the all-1 (all-0) vector (the dimension will be clear from the context).

\subsection{Min-Entropy and Extraction}

The following are information theoretic tools that will be required in our proof. The main notion of entropy used in this work is that of average min-entropy from [DORS08], as well as its smooth version (see Definitions 2.1 and 2.2 below). We then show that applying a pairwise independent hash function with a large enough image on an average min-entropy source, roughly preserves the average min-entropy (that is, it is an entropy condenser). This is derived from the generalized "crooked" leftover hash lemma [DS05b, BFO08].

We start by defining average min-entropy.

Definition 2.1 (average min-entropy [DORS08]). Let $X, Z$ be (possibly dependent) random variables, the average min entropy of $X$ conditioned on $Z$ is:

$$
\widetilde{\mathbf{H}}_{\infty}(X \mid Z)=-\log \left(\underset{z \leftarrow Z}{\mathbb{E}}\left[2^{-\mathbf{H}_{\infty}(X \mid Z=z)}\right]\right)
$$

It follows from the definition that for every deterministic function $f$ (that may depend on $Z$ ):

$$
\widetilde{\mathbf{H}}_{\infty}(f(X) \mid Z) \leq \widetilde{\mathbf{H}}_{\infty}(X \mid Z) .
$$

We will also use a smooth variant introduced in [DORS08, Appendix A] following [RW04].

Definition 2.2 (smooth average min-entropy [DORS08]). Let $X, Z$ be as above and let $\epsilon>0$, then

$$
\widetilde{\mathbf{H}}_{\infty}^{\epsilon}(X \mid Z)=\max _{\left(X^{\prime}, Z^{\prime}\right): \Delta\left((X, Z),\left(X^{\prime}, Z^{\prime}\right)\right) \leq \epsilon} \widetilde{\mathbf{H}}_{\infty}\left(X^{\prime} \mid Z^{\prime}\right)
$$

For our next step, we will require the following tool.

Lemma 2.3 (generalized "crooked" LHL [BFO08, Lemma 7.1]). Let X, Z be random variables, let $\mathcal{H}$ be a pairwise independent hash family, and let $h \leftarrow \mathcal{H}$ be a properly sampled function from this family, finally let $f$ be a function of image size $K$, then

$$
\Delta((f(h(X)), Z, h),(f(U), Z, h)) \leq \frac{1}{2} \sqrt{K} \cdot 2^{-\frac{1}{2} \widetilde{\mathbf{H}}_{\infty}(X \mid Z)},
$$

where $U$ is uniformly distributed in the domain of $f$. 
We will show that pairwise independent function condense average min-entropy in the following way.

Lemma 2.4. Let $X, Z$ be random variables, let $\mathcal{H}$ be a pairwise independent hash family whose output is a binary string of length $\left.\geq \mid \widetilde{\mathbf{H}}_{\infty}(X \mid Z)-2 \log (1 / \epsilon)+2\right\rfloor$, for some $\epsilon>0$. Then letting $h \leftarrow \mathcal{H}$ be a properly sampled function from this family, it holds that

$$
\widetilde{\mathbf{H}}_{\infty}^{\epsilon}(h(X) \mid Z, h) \geq \widetilde{\mathbf{H}}_{\infty}(X \mid Z)-2 \log (1 / \epsilon)+1 .
$$

Proof. Let $X, Z, \mathcal{H}, h, \epsilon$ be as in the lemma statement. Our goal is to show that there exists a random variable $Y$ such that

$$
\Delta((h(X), Z, h),(Y, Z, h)) \leq \epsilon
$$

and

$$
\widetilde{\mathbf{H}}_{\infty}(Y \mid Z, h) \geq \widetilde{\mathbf{H}}_{\infty}(X \mid Z)-2 \log (1 / \epsilon)+1
$$

Let $f$ be the function that outputs the first $k$ bits of its input, for

$$
k=\left\lfloor\widetilde{\mathbf{H}}_{\infty}(X \mid Z)-2 \log (1 / \epsilon)+2\right\rfloor \geq \widetilde{\mathbf{H}}_{\infty}(X \mid Z)-2 \log (1 / \epsilon)+1
$$

and note that $f(U)$ is uniform over $\{0,1\}^{k}$ (in fact, we can use any function that has this property).

Then by Lemma 2.3, it holds that

$$
\Delta((f(h(X)), Z, h),(f(U), Z, h)) \leq \epsilon .
$$

Now, consider a 2-step process for sampling the joint distribution $(h(X), Z, h)$ : first, sample $(f(h(X)), Z, h)$ from the appropriate marginal distribution; and then sample $h(X)$ conditioned on the previously sampled values.

We define $Y$ using using the following process: First, sample a tuple according to the distribution $(f(U), Z, h)$, and then apply the second stage of the sampling process from above. The result will be the distribution $(Y, Z, h)$. Clearly,

$$
\Delta((h(X), Z, h),(Y, Z, h))=\Delta((f(h(X)), Z, h),(\underbrace{f(Y)}_{=f(U)}, Z, h)) \leq \epsilon,
$$

where the first equality is since there is a deterministic mapping $(f)$ from the left hand side to the right hand side, and a randomized mapping (the second step sampler) from the right hand side to the left hand side.

To conclude, we notice that

$$
\widetilde{\mathbf{H}}_{\infty}(Y \mid Z, h) \geq \widetilde{\mathbf{H}}_{\infty}(f(Y) \mid Z, h)=\widetilde{\mathbf{H}}_{\infty}\left(\{0,1\}^{k} \mid Z, h\right)=k \geq \widetilde{\mathbf{H}}_{\infty}(X \mid Z)-2 \log (1 / \epsilon)+1 .
$$

\subsection{Graded Encoding Schemes and Assumptions}

We begin with the definition of a graded encoding scheme, due to Garg, Gentry and Halevi [GGH13]. While their construction is very general, for our purposes a more restricted setting is sufficient as defined below.

Definition 2.5 ( $\tau$-Graded Encoding Scheme [GGH13]). A $\tau$-encoding scheme for a ring $R$ is a collection of sets $\mathcal{S}=\left\{S_{\mathbf{v}}^{(\alpha)} \subset\{0,1\}^{*}: \mathbf{v} \in\{0,1\}^{\tau}, \alpha \in R\right\}$, with the following properties: 
1. For every index $\mathbf{v} \in\{0,1\}^{\tau}$, the sets $\left\{S_{\mathbf{v}}^{(\alpha)}: \alpha \in R\right\}$ are disjoint, and so they are a partition of the indexed set $S_{\mathbf{v}}=\bigcup_{\alpha \in R} S_{\mathbf{v}}^{(\alpha)}$.

2. There are binary operations "+" and "-" such that for all $\mathbf{v} \in\{0,1\}^{\tau}, \alpha_{1}, \alpha_{2} \in R$ and for all $u_{1} \in S_{\mathbf{v}}^{\left(\alpha_{1}\right)}, u_{2} \in S_{\mathbf{v}}^{\left(\alpha_{2}\right)}$ :

$$
u_{1}+u_{2} \in S_{\mathbf{v}}^{\left(\alpha_{1}+\alpha_{2}\right)} \text { and } \quad u_{1}-u_{2} \in S_{\mathbf{v}}^{\left(\alpha_{1}-\alpha_{2}\right)},
$$

where $\alpha_{1}+\alpha_{2}$ and $\alpha_{1}-\alpha_{2}$ are addition and subtraction in $R$.

3. There is an associative binary operation " $\times$ " such that for all $\mathbf{v}_{1}, \mathbf{v}_{2} \in\{0,1\}^{\tau}$ such that $\mathbf{v}_{1}+\mathbf{v}_{2} \in\{0,1\}^{\tau}$, for all $\alpha_{1}, \alpha_{2} \in R$ and for all $u_{1} \in S_{\mathbf{v}_{1}}^{\left(\alpha_{1}\right)}, u_{2} \in S_{\mathbf{v}_{2}}^{\left(\alpha_{2}\right)}$, it holds that

$$
u_{1} \times u_{2} \in S_{\mathbf{v}_{1}+\mathbf{v}_{2}}^{\left(\alpha_{1} \cdot \alpha_{2}\right)},
$$

where $\alpha_{1} \cdot \alpha_{2}$ is multiplication in $R$.

In this work, the ring $R$ will always be $\mathbb{Z}_{p}$ for a prime $p$.

To the reader who is familiar with the [GGH13] work, we note that the above is the special case of the [GGH13] construction in which we consider only binary index vectors (in the [GGH13] notation, this corresponds to setting $\kappa=1$ ), and we construct our encoding schemes to be asymmetric (as will become apparent below when we define our zero-text index vzt $=\overrightarrow{1}$ ).

Definition 2.6 (Efficient Procedures for a $\tau$-Graded Encoding Scheme [GGH13]). We consider $\tau$-graded encoding schemes (see above) where the following procedures are efficiently computable.

- Instance Generation: InstGen $\left(1^{\lambda}, 1^{\tau}\right)$ outputs the set of parameters params, a description of a $\tau$-Graded Encoding Scheme. (Recall that we only consider Graded Encoding Schemes over the set indices $\{0,1\}^{\tau}$, with zero testing in the set $S_{\overrightarrow{1}}$ ). In addition, the procedure outputs a subset evparams $\subset$ params that is sufficient for computing addition, multiplication and zero testing ${ }^{6}$ (but possibly insufficient for encoding or for randomization).

- Ring Sampler: samp(params) outputs a "level zero encoding" $a \in S_{0}^{(\alpha)}$ for a nearly uniform $\alpha \in_{R} R$.

- Encode and Re-Randomize: ${ }^{7}$ encRand(params, $\left.i, a\right)$ takes as input an index $i \in[\tau]$ and $a \in S_{0}^{(\alpha)}$, and outputs an encoding $u \in S_{\mathbf{e}_{i}}^{(\alpha)}$, where the distribution of $u$ is (statistically close to being) only dependent on $\alpha$ and not otherwise dependent of $a$.

- Addition and Negation: add(evparams, $\left.u_{1}, u_{2}\right)$ takes $u_{1} \in S_{\mathbf{v}}^{\left(\alpha_{1}\right)}, u_{2} \in S_{\mathbf{v}}^{\left(\alpha_{2}\right)}$, and outputs $w \in S_{\mathbf{v}}^{\left(\alpha_{1}+\alpha_{2}\right)}$. (If the two operands are not in the same indexed set, then add returns $\perp$ ). We often use the notation $u_{1}+u_{2}$ to denote this operation when evparams is clear from the context. Similarly, negate(evparams, $\left.u_{1}\right) \in S_{\mathbf{v}}^{\left(-\alpha_{1}\right)}$.

\footnotetext{
${ }^{6}$ The "zero testing" parameter pzt defined in [GGH13] is a part of evparams.

${ }^{7}$ This functionality is not explicitly provided by [GGH13], however it can be obtained by combining their encoding and re-randomization procedures.
} 
- Multiplication: mult(evparams, $\left.u_{1}, u_{2}\right)$ takes $u_{1} \in S_{\mathbf{v}_{1}}^{\left(\alpha_{1}\right)}, u_{2} \in S_{\mathbf{v}_{2}}^{\left(\alpha_{2}\right)}$. If $\mathbf{v}_{1}+\mathbf{v}_{2} \in\{0,1\}^{\tau}$ (i.e. every coordinate in $\mathbf{v}_{1}+\mathbf{v}_{2}$ is at most 1$)$, then mult outputs $w \in S_{\mathbf{v}_{1}+\mathbf{v}_{2}}^{\left(\alpha_{2} \cdot \alpha_{2}\right)}$. Otherwise, mult outputs $\perp$. We often use the notation $u_{1} \times u_{2}$ to denote this operation when evparams is clear from the context.

- Zero Test: isZero(evparams, $u$ ) outputs 1 if $u \in S_{\overrightarrow{1}}^{(0)}$, and 0 otherwise.

In the [GGH13, CLT13] constructions, encodings are noisy and the noise level increases with addition and multiplication operations, so one has to be careful not to go over a specified noise bound. However, the parameters can be set so as to support $O(\tau)$ operations, which are sufficient for our purposes. We therefore ignore noise management throughout this manuscript. An additional subtle issue is that with negligible probability the initial noise may be too big. However this can be avoided by adding rejection sampling to samp and therefore ignored throughout the manuscript as well.

It is important to notice that our definition deviates from that of [GGH13] as we define two sets of parameters params and evparams. While the former will be used by the obfuscator in our construction (and therefore will not be revealed to an external adversary), the latter will be used when evaluating an obfuscated program (and thus will be known to an adversary). When instantiating our definition, the guideline is to make evparams minimal so as to give the least amount of information to the adversary. In particular, in the known candidates [GGH13, CLT13], evparams only needs to contain the zero-test parameter pzt (as well as the global modulus).

\subsubsection{Hardness Assumptions}

In this work, we will use two hardness assumptions over graded encoding schemes. The first, which we call "graded external DDH" (or GXDH, Assumption 2.7 below) is an analog of the symmetric external DH assumption (SXDH), instantiated for the multilinear case. The second assumption (GCAN Assumption 2.8) is an analog of Canetti's assumption [Can97], that taking a random generator to a high-entropy power results in a random-looking element. We note that we make these assumptions against non-uniform adversaries.

Assumption 2.7 (Graded External DH). Let (params, evparams) $\leftarrow \operatorname{InstGen}\left(1^{\lambda}, 1^{\tau}\right)$, for all $i=1, \ldots, \tau$, sample $r_{i, 0}, r_{i, 1}, a_{i, 0}, a_{i, 1} \leftarrow \operatorname{samp}($ params $)$ and consider the following values:

$$
\begin{array}{ll}
w_{i, 0} \leftarrow \operatorname{encRand}\left(\text { params }, i, r_{i, 0}\right) & w_{i, 1} \leftarrow \operatorname{encRand}\left(\text { params }, i, r_{i, 1}\right) \\
u_{i, 0} \leftarrow \operatorname{encRand}\left(\text { params }, i, r_{i, 0} \times a_{i, 0}\right) & u_{i, 1} \leftarrow \operatorname{encRand}\left(\text { params }, i, r_{i, 1} \times a_{i, 1}\right) \\
& u_{i, 1}^{\prime} \leftarrow \operatorname{encRand}\left(\text { params }, i, r_{i, 1} \times a_{i, 0}\right)
\end{array}
$$

The GXDH assumption is that for every choice of $\tau \in \mathbb{N}$ and $i^{*} \in[\tau]$, no ensemble of polynomial time adversaries can have have non-negligible advantage in distinguishing the distributions:

$$
\begin{gathered}
\text { (evparams, } \left.\left\{\left(w_{i, 0}, u_{i, 0}, w_{i, 1}, u_{i, 1}, u_{i, 1}^{\prime}\right)\right\}_{i \neq i^{*}},\left(w_{i^{*}, 0}, u_{i^{*}, 0}, w_{i^{*}, 1}, u_{i^{*}, 1}\right)\right) \\
\text { and } \\
\left(\text { evparams, }\left\{\left(w_{i, 0}, u_{i, 0}, w_{i, 1}, u_{i, 1}, u_{i, 1}^{\prime}\right)\right\}_{i \neq i^{*}},\left(w_{i^{*}, 0}, u_{i^{*}, 0}, w_{i^{*}, 1}, u_{i^{*}, 1}^{\prime}\right)\right)
\end{gathered}
$$


We note that a stronger version of this assumption, where the distinguisher is given access to params rather than evparams, was presented in the the early versions of [GGH13]. It was later shown that this stronger assumption is false, see later versions of [GGH13] for the attack. We emphasize that no attacks are known if only evparams is given as above. Furthermore, the new candidate of [CLT13] is not known to be sensitive to such attacks even if params is given.

Since we only provide our distinguisher with evparams, it may not be able to generate DDH tuples by itself. We therefore provide it with correctly labeled DDH samples for all groups except $i^{*}$. This is the minimal assumption that is required for our construction, however we conjecture that a stronger variant where the adversary is allowed to receive an unbounded number of labeled samples at any group (including $i^{*}$ ) is also true.

For our next assumption, we introduce the following notation. Consider a distribution $D$ over $S_{\mathbf{v}}=\cup_{\alpha \in R} S_{\mathbf{v}}^{(\alpha)}$. The distribution enc ${ }^{-1}(D)$ is defined by the following process: Sample $x \leftarrow D$, let $\alpha$ be such that $x \in S_{\mathbf{v}}^{(\alpha)}$, output $\alpha$. We also recall the definition of smooth average min-entropy (see Definition 2.2 above).

Assumption 2.8 ("Graded Canetti"). Let (params, pzt) $\leftarrow \operatorname{lnstGen}\left(1^{\lambda}, 1^{\tau}\right)$ and let $\left\{\left(D_{\lambda}, Z_{\lambda}\right)\right\}_{\lambda \in \mathbb{N}}$ be a distribution ensemble over $S_{0} \times\{0,1\}^{*}$, such that

$$
\widetilde{\mathbf{H}}_{\infty}^{\epsilon}\left(\text { enc }^{-1}\left(D_{\lambda}\right) \mid Z_{\lambda}\right) \geq h(\lambda)
$$

for some $\epsilon=\operatorname{negl}(\lambda)$ and function $h(\lambda)=\omega(\log \lambda)$.

The GCAN assumption is that no ensemble of polynomial time adversaries and indices $i$ can have non-negligible advantage in distinguishing the distributions

$$
\text { (params, evparams, } \left.w, u, z) \text { and (params, evparams, } w, u^{\prime}, z\right) \text {, }
$$

where: $($ params, evparams $) \leftarrow \operatorname{InstGen}\left(1^{\lambda}, 1^{\tau}\right), r \leftarrow \operatorname{samp}($ params $), w \leftarrow \operatorname{encRand}($ params $, i, r)$, $(x, z) \leftarrow\left(D_{\lambda}, Z_{\lambda}\right), u \leftarrow$ encRand (params, $\left.i, r \times x\right), u^{\prime} \leftarrow$ encRand(params, $i$, samp (params)). (Note that in this definition, the distinguisher is given both params and evparams.)

This assumption is consistent with out knowledge on candidate graded encoding schemes. However, if we want to make an even weaker assumption, we can set the minimal entropy requirement to be higher than just $\omega(\log \lambda)$. The constructions in this paper can trivially be adapted to such weaker variants (with the expected degradation in security).

\subsection{Obfuscation}

Definition 2.9 (Virtual Black-Box Obfuscator $\left[\mathrm{BGI}^{+} 12\right]$ ). Let $\mathcal{C}=\left\{\mathcal{C}_{n}\right\}_{n \in \mathbb{N}}$ be a family of polynomial-size circuits, where $\mathcal{C}_{n}$ is a set of boolean circuits operating on inputs of length $n$. And let $\mathcal{O}$ be a PPTM algorithm, which takes as input an input length $n \in \mathbb{N}$, a circuit $C \in \mathcal{C}_{n}$, a security parameter $\lambda \in \mathbb{N}$, and outputs a boolean circuit $\mathcal{O}(C)$ (not necessarily in $\mathcal{C}$ ).

$\mathcal{O}$ is an obfuscator for the circuit family $\mathcal{C}$ if it satisfies:

1. Preserving Functionality: For every $n \in \mathbb{N}$, and every $C \in \mathcal{C}_{n}$, and every $\vec{x} \in\{0,1\}^{n}$, with all but $\operatorname{negl}(\lambda)$ probability over the coins of $\mathcal{O}$ :

$$
\left(\mathcal{O}\left(C, 1^{n}, \lambda\right)\right)(\vec{x})=C(\vec{x})
$$


2. Polynomial Slowdown: For every $n, \lambda \in \mathbb{N}$ and $C \in \mathcal{C}$, the circuit $\mathcal{O}\left(C, 1^{n}, 1^{\lambda}\right)$ is of size at most poly $(|C|, n, \lambda)$.

3. Virtual Black-Box: For every (non-uniform) polynomial size adversary $\mathcal{A}$, there exists a (nonuniform) polynomial size simulator $\mathcal{S}$, such that for every $n \in \mathbb{N}$ and for every $C \in \mathcal{C}_{n}$ :

$$
\left|\operatorname{Pr}_{\mathcal{O}, \mathcal{A}}\left[\mathcal{A}\left(\mathcal{O}\left(C, 1^{n}, 1^{\lambda}\right)\right)=1\right]-\operatorname{Pr}_{\mathcal{S}}\left[\mathcal{S}^{C}\left(1^{|C|}, 1^{n}, 1^{\lambda}\right)=1\right]\right|=\operatorname{negl}(\lambda)
$$

Remark 2.10. A stronger notion of functionality, which also appears in the literature, requires that with overwhelming probability the obfuscated circuit is correct on every input simultaneously. We use the relaxed requirement that for every input (individually) the obfuscated circuit is correct with overwhelming probability (in both cases the probability is only over the obfuscator's coins). We note that our construction can be modified to achieve the stronger functionality property (by using a ring of sufficiently large size and the union bound).

Definition 2.11 (Average-Case Secure Virtual Black-Box). Let $\mathcal{C}=\left\{\mathcal{C}_{n}\right\}_{n \in \mathbb{N}}$ be a family of circuits and $\mathcal{O}$ a PPTM as in Definition 2.9. Let $\mathcal{D}=\left\{\mathcal{D}_{n}\right\}_{n \in \mathbb{N}}$ be an ensemble of distribution families $\mathcal{D}_{n}$, where each $D \in \mathcal{D}_{n}$ is a distribution over $\mathcal{C}_{n}$.

$\mathcal{O}$ is an obfuscator for the distribution class $\mathcal{D}$ over the circuit family $\mathcal{C}$, if it satisfies the functionality and polynomial slowdown properties of Definition 2.9 with respect to $\mathcal{C}$, but the virtual black-box property is replaced with:

3. Distributional Virtual Black-Box: For every (non-uniform) polynomial size adversary $\mathcal{A}$, there exists a (non-uniform) polynomial size simulator $\mathcal{S}$, such that for every $n \in \mathbb{N}$, every distribution $D \in \mathcal{D}_{n}$ (a distribution over $\mathcal{C}_{n}$ ), and every predicate $P: C_{n} \rightarrow\{0,1\}$ :

$$
\left|\underset{C \sim D_{n}, \mathcal{O}, \mathcal{A}}{\operatorname{Pr}}\left[\mathcal{A}\left(\mathcal{O}\left(C, 1^{n}, 1^{\lambda}\right)\right)=P(C)\right]-\operatorname{Pr}_{C \sim D_{n}, \mathcal{S}}\left[\mathcal{S}^{C}\left(1^{|C|}, 1^{n}, 1^{\lambda}\right)=P(C)\right]\right|=\operatorname{negl}(\lambda)
$$

Remark 2.12. Our proof of average-case security for the conjunction obfuscator (Theorem 4.2) is in fact stronger. We show a simulator $\mathcal{S}$ that does not even require black-box access to the circuit $C$. Rather, for a circuit $C$ drawn from a distribution in $\mathcal{D}$, the probability of predicting $P(C)$ from an obfuscation of $C$, is the same as the probability of predicting $P(C)$ from a "dummy obfuscation" that is independent of $C$. See the proof for further details.

\section{Obfuscating Conjunctions}

In this section we present our obfuscator for conjunctions ConjObf (Figure 1). We provide a proof of security for functions that are not determined by the locations of the wildcards in Section 4 . Finally, in Section 5 we provide evidence of the security of our construction for any conjunction, by proving that it is secure against generic adversaries that do not use the representation of the specific graded encoding scheme.

We start with a formal definition of the class of conjunctions.

Definition 3.1 (n-bit Conjunction). For an input length $n$, a conjunction $C=(W, V):\{0,1\}^{n} \rightarrow$ $\{0,1\}$ is a predicate on $n$-bit inputs, which is defined by two vectors $W, V \in\{0,1\}^{n}$. For every input $\vec{x} \in\{0,1\}^{n}, C(\vec{x})=1$ iff for all $i \in[n], W[i]=1$ or $V[i]=\vec{x}[i]$. For the sake of unity of representation, we require that whenever $W[i]=1$, it holds that $V[i]=0$. 
We often alternate between treating $W$ as an index vector and treating it as a subset of $[n]$. If $i \in W$ then we say that $i$ is a wildcard location.

An ensemble of conjunctions is defined in the standard way.

Definition 3.2 (Conjunction Ensemble). A conjunction ensemble $\mathcal{C}=\left\{C_{n}\right\}_{n \in \mathbb{N}}$ is a collection of conjunctions $C_{n}:\{0,1\}^{n} \rightarrow\{0,1\}$, one for each input length.

We now state a useful (for our purposes) property of conjunctions:

Claim 3.3. There exists an efficient algorithm $\mathcal{B}$, such that for any conjunction $C=(W, V)$, and any accepting input $\vec{x}$ of $C, \mathcal{B}$ can recover $(W, V)$ :

$$
\forall C=(W, V), \forall \vec{x}: C(\vec{x})=1, \quad \mathcal{B}^{C}(\vec{x})=(W, V)
$$

Proof. Take $n=|\vec{x}|$, the algorithm $\mathcal{B}$ enumerates over the bits of $\vec{x}$. For each bit $i$, it flips the $i$-th bit of $\vec{x}: \vec{x}^{(i)}=\vec{x} \oplus \mathbf{e}_{i}$, and checks whether $C\left(\vec{x}^{(i)}\right)=1$. If so, then $i$ must be a wildcard: $W[i]=1$ and $V[i]=0$. Otherwise, $i$ is not a wildcard: $W[i]=0$ and $V[i]=\vec{x}[i]$.

Our obfuscator for the class of conjunctions is presented in Figure 1. Correctness follows in a straightforward manner as described in the following lemma (the proof is omitted). We note that the error is one sided, it is always the case that if $C(\vec{x})=1$ then for the obfuscated program $\mathcal{O}_{C}(\vec{x})=1$ as well.

Lemma 3.4 (Obfuscator Functionality). Let $C$ be an $n$-variable conjunction and consider its obfuscation $\mathcal{O}_{C}=\operatorname{ConjObf}\left(C, 1^{n}, 1^{\lambda}\right)$. Then for all $\vec{x}$,

$$
\operatorname{Pr}\left[\mathcal{O}_{C}(\vec{x}) \neq C(\vec{x})\right] \leq \operatorname{poly}(n) / p,
$$

where $p=2^{\Omega(\lambda)}$ is the order of the group in the graded encoding scheme, and the probability is taken over the randomness of ConjObf.

As a concluding remark, we note that if our graded encoding scheme has the property that $p \gg 2^{n}$ (which is indeed achievable in the candidate of [GGH13]), then a stronger correctness guarantee, as mentioned in Remark 2.10, can be achieved by using the union bound. In this parameter range, the proof of security also becomes somewhat simpler (see Section 4). However, we want to present our scheme in the most generic way so as to be compatible with possible choices of the security parameter and with future graded encoding schemes.

\section{Security from GXDH and GCAN}

In this section we prove that ConjObf is a secure distributional black box obfuscator for any distribution over the conjunctions family for which the function is hard to determine (i.e. has superlogarithmic entropy) even if the locations of all the wildcards are known. Namely, there is sufficient min-entropy in $V$ even given $W$ (recall that $V[i]=0$ wherever $W[i]=1$ ).

Definition 4.1 (equivocality given wildcards). Let $\mathcal{C}$ be the class of conjunctions, and let $\mathcal{D}=\left\{\mathcal{D}_{\lambda}\right\}$ be an ensemble of families of distributions. We say that $\mathcal{D}$ is equivocal given the wildcards if there exists $h(\lambda)=\omega(\log \lambda)$ such that for all $D \in \mathcal{D}_{\lambda}$, if $(V, W) \leftarrow D$ then

$$
\widetilde{\mathbf{H}}_{\infty}(V \mid W) \geq h(\lambda) .
$$


Obfuscator ConjObf, on input $\left(1^{\lambda}, 1^{n}, C=(W, V)\right)$

1. generate $($ params, evparams $) \leftarrow \operatorname{InstGen}\left(1^{\lambda}, 1^{n+1}\right)$

2. for $i \in[n]$ :

if $i \in W$, then: $a_{i, 0}=a_{i, 1} \leftarrow \operatorname{samp}($ params $) \in S_{0}^{\left(\alpha_{i, 0}\right)}=S_{0}^{\left(\alpha_{i, 1}\right)}$

if $i \notin W$, then: $a_{i, 0} \leftarrow \operatorname{samp}($ params $) \in S_{0}^{\left(\alpha_{i, 0}\right)}, a_{i, 1} \leftarrow \operatorname{samp}($ params $) \in S_{0}^{\left(\alpha_{i, 1}\right)}$

3. for $i \in[n]$ :

$$
\begin{aligned}
& r_{i, 0} \leftarrow \operatorname{samp}(\text { params }) \in S_{0}^{\left(\rho_{i, 0}\right)}, r_{i, 1} \leftarrow \operatorname{samp}(\text { params }) \in S_{0}^{\left(\rho_{i, 0}\right)} \\
& s_{i, 0} \leftarrow r_{i, 0} \times a_{i, 0} \in S_{0}^{\left(\rho_{i, 0} \cdot \alpha_{i, 0}\right)}, s_{i, 1} \leftarrow r_{i, 1} \times a_{i, 1} \in S_{0}^{\left(\rho_{i, 1} \cdot \alpha_{i, 1}\right)} \\
& w_{i, 0} \leftarrow \operatorname{encRand}\left(\text { params }, i, r_{i, 0}\right) \in S_{\mathbf{e}_{i}}^{\left(\rho_{i, 0}\right)}, w_{i, 1} \leftarrow \operatorname{encRand}\left(\text { params }, i, r_{i, 1}\right) \in S_{\mathbf{e}_{i}}^{\left(\rho_{i, 1}\right)} \\
& u_{i, 0} \leftarrow \operatorname{encRand}\left(\text { params }, i, s_{i, 0}\right) \in S_{\mathbf{e}_{i}}^{\left(\rho_{i, 0} \cdot \alpha_{i, 0}\right)}, u_{i, 1} \leftarrow \operatorname{encRand}\left(\text { params }, i, s_{i, 1}\right) \in S_{\mathbf{e}_{i}}^{\left(\rho_{i, 1} \cdot \alpha_{i, 1}\right)} \\
4 . & \left.a_{n+1} \leftarrow\left(\Pi_{i \in[n]} a_{i, V[i]}\right) \in S_{0}^{\left(\Pi_{i \in[n]} \alpha_{i, V}[i]\right.}\right) \\
& r_{n+1} \leftarrow \operatorname{samp}(\text { params }) \in S_{0}^{\left(\rho_{n+1}\right)} \\
& s_{n+1} \leftarrow r_{n+1} \times a_{n+1} \in S_{0}^{\left(\rho_{n+1} \cdot \Pi_{i \in[n]} \alpha_{i, V[i]}\right)} \\
& w_{n+1} \leftarrow \operatorname{encRand}\left(\text { params }, n+1, r_{n+1}\right) \in S_{\mathbf{e}_{n+1}}^{\left(\rho_{n+1}\right)} \\
& u_{n+1} \leftarrow \operatorname{encRand}\left(\text { params }, n+1, s_{n+1}\right) \in S_{\mathbf{e}_{n+1}}^{\left(\rho_{n+1} \cdot \Pi_{i \in[n]} \alpha_{i, V[i]}\right)}
\end{aligned}
$$

5. output the obfuscation: (evparams, $\left.\left\{\left(w_{i, 0}, u_{i, 0}\right),\left(w_{i, 1}, u_{i, 1}\right)\right\}_{i \in[n]},\left(w_{n+1}, u_{n+1}\right)\right)$

Evaluation, on input $\vec{x} \in\{0,1\}^{n}$

1. $\mathbf{t} \leftarrow\left(w_{n+1} \times \Pi_{i \in[n]} u_{i, \vec{x}[i]}\right) \in S_{(1,1, \ldots, 1)}^{\left(\rho_{n+1} \cdot\left(\Pi_{i \in[n]} \rho_{i, \vec{x}[i]} \cdot \alpha_{i, \vec{x}[i]}\right)\right)}$

2. $\mathbf{t}^{\prime} \leftarrow\left(u_{n+1} \times \Pi_{i \in[n]} w_{i}\right) \in S_{(1,1, \ldots, 1)}^{\left(\rho_{n+1} \cdot\left(\Pi_{i \in[n]} \alpha_{i, V[i]}\right) \cdot\left(\Pi_{i \in[n]} \rho_{i, \vec{x}[i]}\right)\right)}$

3. output the bit: isZero(evparams, $\left.\left(\mathbf{t}-\mathbf{t}^{\prime}\right)\right)$.

Figure 1: Obfuscator for Conjunctions.

We will prove the security of ConjObf for such functions under the GXDH and GCAN assumptions (see Section 2.2).

Theorem 4.2. Based on the GXDH and GCAN assumptions, the algorithm ConjObf is an average-case black-box obfuscator for ensembles of distribution families that are equivocal given the wildcards.

Proof. We start by stating a claim that will be used later on in the proof.

Claim 4.3. Let $p$ be prime and let $k \in \mathbb{N}$ be integer. Consider the hash family $\mathcal{H} \subseteq\{0,1\}^{k} \rightarrow \mathbb{Z}_{p}^{*}$, where each function in $\mathcal{H}$ is defined by a sequence $a_{0}, a_{1}, \ldots, a_{k} \in \mathbb{Z}_{p}^{*}$ and

$$
\mathcal{H}_{a_{0}, a_{1}, \ldots, a_{k}}\left(x_{1}, \ldots, x_{k}\right)=a_{0} \cdot \prod_{i \in[k]} a_{i}^{x_{i}},
$$

then $\mathcal{H}$ is pairwise independent. 
This claim follows in a straightforward manner since $\mathcal{H}$ (defined therein) is a random linear function "in the exponent".

Consider a function $C=(W, V)$ drawn from a distribution $\mathcal{D}_{\lambda}$, and consider the distribution of a properly obfuscated program $\operatorname{ConjObf}(C)$. We will show, using a sequence of hybrids, that this distribution is computationally indistinguishable from one that does not depend on $C$, even for a distinguisher who knows the value of the predicate $P(C)$. This will immediately imply a simulator. We note that our proof works even for $P(C)$ with multiple-bit output, so long as $h(\lambda)-|P(C)|=\omega(\log \lambda)$.

1. In this hybrid, we use ConjObf as prescribed:

$$
\mathcal{O}_{C}=\operatorname{ConjObf}(C)=\left(\text { params, pzt },\left\{\left(w_{i, b}, u_{i, b}\right)\right\}_{i \in[n], b \in\{0,1\}},\left(w_{n+1}, u_{n+1}\right)\right) .
$$

2. We change the algorithm so that $a_{i, b} \notin S_{0}^{(0)}$. This is implemented efficiently by rejection sampling, using the zero-test procedure. In this hybrid, therefore, $\alpha_{i, b}$ is uniform in $\mathbb{Z}_{p}^{*}$.

This hybrid only incurs a negligible $\operatorname{poly}(\lambda) / p$ statistical distance in the distribution of $\mathcal{O}_{C}$ compared to the previous hybrid.

3. We change step 4 of the obfuscator. In particular, we will now sample $a_{n+1} \leftarrow \operatorname{samp}($ params $)$ (conditioned on it not being zero, as above). This means that $a_{n+1} \in S_{0}^{\left(\alpha_{n+1}\right)}$ for a random $\alpha_{n+1} \in \mathbb{Z}_{p}^{*}$.

We will now show that the resulting $\mathcal{O}_{C}$ distribution is computationally indistinguishable from the previous hybrid under the GCAN assumption (Assumption 2.8), even when the distinguisher knows $P(C)$. Namely, we will show that for some negligible $\epsilon$, the distributions in the previous hybrid are such that

$$
\widetilde{\mathbf{H}}_{\infty}^{\epsilon}\left(\alpha_{n+1} \mid\left\{\left(w_{i, b}, u_{i, b}\right)\right\}_{i \in[n], b \in\{0,1\}}, P(C)\right)=\omega(\log \lambda)
$$

which will allow us to apply GCAN and conclude that $\alpha_{n+1}$ can be replaced by a uniform variable.

To show that Eq. (5) holds, we present a slightly different way to generate the variables $\alpha_{i, b}$ (note that from this point and on, we are a completely information-theoretic setting, so we will not worry about computational aspects). We will first sample $\left\{\hat{\alpha}_{i, b}\right\}_{i \in[n], b \in\{0,1\}}$ completely uniformly in $\mathbb{Z}_{p}^{*}$, and then set $\alpha_{i, b}$ as follows. If $W[i]=0$ then $\alpha_{i, 0}=\hat{\alpha}_{i, 0}, \alpha_{i, 1}=\hat{\alpha}_{i, 1}$; and if $W[i]=1$ then $\alpha_{i, 0}=\alpha_{i, 1}=\hat{\alpha}_{i, 0}$. Note that the resulting distribution of the $\alpha$ 's is exactly as prescribed. Further notice that

$$
\alpha_{n+1}=\prod_{i \in[n]} \alpha_{i, 0}^{1-V[i]} \alpha_{i, 1}^{V[i]}=\prod_{i \in[n]} \hat{\alpha}_{i, 0}^{1-V[i]} \hat{\alpha}_{i, 1}^{V[i]}=\prod_{i \in[n]} \hat{\alpha}_{i, 0} \cdot \prod_{i \in[n]}\left(\hat{\alpha}_{i, 1} / \hat{\alpha}_{i, 0}\right)^{V[i]}
$$

where the second equality is since $\alpha$ and $\hat{\alpha}$ only differ where $W[i]=V[i]=0$. By Claim 4.3 it follows, therefore, that $\alpha_{n+1}$ is the output of a pairwise-independent hash function applied to $V$.

We proceed to apply Lemma 2.4. We note that $\widetilde{\mathbf{H}}_{\infty}(V \mid W, P(C)) \geq \widetilde{\mathbf{H}}_{\infty}(V \mid W)-|P(C)| \geq$ $h(\lambda)-1$. Therefore there must exist $h^{\prime}(\lambda)=\omega(\log \lambda)$ such that $h^{\prime}(\lambda) \leq h(\lambda)-1$, and in 
addition the length of $\alpha_{n+1}$ is at least $h^{\prime}(\lambda) / 3+2$. We can thus apply Lemma 2.4 with $\epsilon=2^{-h^{\prime}(\lambda) / 3}=\operatorname{negl}(\lambda)$ to argue that

$$
\widetilde{\mathbf{H}}_{\infty}^{\epsilon}\left(\alpha_{n+1} \mid W, P(C),\left\{\hat{\alpha}_{i, b}\right\}\right) \geq h^{\prime}(\lambda) / 3=\omega(\log \lambda) .
$$

Finally, Eq. (5) follows by noticing that there is an invertible mapping between $W,\left\{\hat{\alpha}_{i, b}\right\}$ and $\left\{\left(w_{i, b}, u_{i, b}\right)\right\}_{i \in[n], b \in\{0,1\}}$.

It is interesting to note that this hybrid (and therefore our entire argument) works not only for predicates. In fact, $\ell$-bit functions of the circuit $C$ can be used, so long as $h(\lambda)-\ell=\omega(\log \lambda)$.

At this point, $\mathcal{O}_{C}$ does not depend on $V$ anymore, however it still depends on $W$ via step 2 of ConjObf.

4. We again allow $a_{i, b}$ to be zero. As above, the statistical difference is $\operatorname{poly}(\lambda) / p=n e g l(\lambda)$.

5. We change step 2 of the obfuscator to always act as if $i \notin W$, namely $\alpha_{i, 0}$ and $\alpha_{i, 1}$ are uniform and independent.

A sequence of $n$ hybrids will show that any adversary distinguishing this distribution from the previous one, can be used to break GXDH with only a factor $n$ loss in the advantage. This implies that the hybrids are computationally indistinguishable assuming GXDH. Note that knowledge of $P(C)$ (or even of $C$ in its entirety) is useless for the distinguisher at this point.

After the last hybrid, we are at a case where all $a_{i, b}, r_{i, b}, a_{n+1}, r_{n+1}$ are completely independent of each other, and are sampled in the same way regardless of $(V, W)$. It follows that our final distribution is independent of $C$, but produces $\mathcal{O}_{C}$ indistinguishable from $\operatorname{ConjObf}(C)$ (even given $P(C)$ ). Since this distribution is efficiently sampleable (via the process we describe in the proof), the theorem follows.

\section{Security in the Generic Graded Encoding Scheme (GES) Model}

\subsection{The Generic Graded Encoding Scheme Model}

We would like to prove the security of our construction against generic adversaries. To this end, we will use the generic graded encoding scheme model, analogous to the generic group model (see Shoup [Sho97] and Maurer [Mau05]). In this model, an algorithm/adversary $\mathcal{A}$ can only interact with the graded encoding scheme via oracle calls to the add, mult, and isZero operations from Definition 2.6. Note that, in particular, we only allow access to the operations that can be run using evparams. To the best of our knowledge, non-generic attacks on known schemes require use of params and cannot be mounted when only evparams is given.

We use $\mathcal{G}$ to denote an oracle that answers adversary calls. The oracle operates as follows: for each index $\mathbf{v} \in\{0,1\}^{\tau}$, the elements of the indexed set $S_{\mathbf{v}}=\bigcup_{\alpha \in R} S_{\mathbf{v}}^{(\alpha)}$ are arbitrary binary strings. The adversary $\mathcal{A}$ can manipulate these strings using oracle calls (via $\mathcal{G}$ ) to the graded encoding scheme's functionalities. For example, the adversary can use $\mathcal{G}$ to perform an add call: taking strings $s_{1} \in S_{\mathbf{v}}^{\left(\alpha_{1}\right)}, s_{2} \in S_{\mathbf{v}}^{\left(\alpha_{2}\right)}$, encoding indexed ring elements $\left(\mathbf{v}, \alpha_{1}\right),\left(\mathbf{v}, \alpha_{2}\right)$ (respectively), and obtaining a string $s \in S_{\mathbf{v}}^{\left(\alpha_{1}+\alpha_{2}\right)}$, encoding the indexed ring element $\left(\mathbf{v},\left(\alpha_{1}+\alpha_{2}\right)\right)$. 
We say that $\mathcal{A}$ is a generic algorithm (or adversary) for a problem on graded encoding schemes (e.g. for computing a moral equivalent of discreet log), if it can accomplish this task with respect to any oracle representing a graded encoding scheme, see below.

In the add example above, there may be many strings/encodings in the set $S_{\mathbf{v}}^{\left(\alpha_{1}+\alpha_{2}\right)}$. One immediate question is which of these elements should be returned by the call to add. In our abstraction, for each $\mathbf{v} \in\{0,1\}^{\tau}$ and $\alpha \in R, \mathcal{G}$ always uses a single unique encoding of the indexed ring element $(\mathbf{v}, \alpha)$. I.e. the set $S_{\mathbf{v}}^{\alpha}$ is a singleton. Thus, the representation of items in the graded encoding scheme is given by a map $\sigma(\mathbf{v}, \alpha)$ from $\mathbf{v} \in\{0,1\}^{\tau}$ and $\alpha \in R$, to $\{0,1\}^{*}$. We restrict our attention to the case where this mapping has polynomial blowup.

Remark 5.1 (Unique versus Randomized Representation). We note that the known candidate of secure graded encoding schemes [GGH13] does not provide unique encodings: their encodings are probabilistic. Nonetheless, in the generic graded encoding scheme abstraction we find it helpful to restrict our attention to schemes with unique encodings. For the purposes of proving security against generic adversaries, this makes sense: a generic adversary should work for any implementation of the oracle $\mathcal{G}$, and in particular also for an implementation that uses unique encodings.

Moreover, our perspective is that unique encodings are more "helpful" to an adversary than randomized encodings: a unique encoding gives the adversary the additional power to "automatically" check whether two encodings are of the same indexed ring element (without consulting the oracle). Thus, we prefer to prove security against generic adversaries even for unique representations.

We remark that the set of legal encodings may be very sparse within the set of images of $\sigma$, and indeed this is the main setting we will consider when we study the generic model. In this case, the only way for $\mathcal{A}$ to obtain a valid representation of any element in any graded set is via calls to the oracle. Finally, we note that if oracle calls contain invalid operators (e.g. the input is not an encoding of an element in any graded set, the inputs to add are not in the same graded set, etc.), then the oracle returns $\perp$.

Random Graded Encoding Scheme Oracle. We focus on a particular randomized oracle: the random generic encoding scheme (GES) oracle $\mathcal{R G}$. $\mathcal{R G}$ operates as follows: for each indexed ring element (with index $\mathbf{v} \in\{0,1\}^{\tau}$ and ring element $\sigma \in R$ ), its encoding is of length $\ell=$ $(\tau \cdot \log |R| \cdot \operatorname{poly}(\lambda))$. The encoding of each indexed ring element is a uniformly random string of length $\ell$. In particular, this implies that the only way that $\mathcal{A}$ can obtain valid encodings is by calls to the oracle $\mathcal{R} \mathcal{G}$ (except with negligible probability).

The oracle $\mathcal{R G}$ maintains a table $T$ (initially empty) containing the indexed ring elements that were provided as outputs to prior calls made by $\mathcal{A}$. As the execution of $\mathcal{A}$ proceeds, its oracle calls are handled one at a time. On each such call, the oracle $\mathcal{R} \mathcal{G}$ checks whether every input argument that encodes an indexed ring element is the output of a previous call to $\mathcal{R} \mathcal{G}$. If not, then the oracle immediately returns $\perp$ (at least one input argument is an invalid encoding). Otherwise, the oracle retrieves from its table $T$ the indexed ring elements encoded in all input arguments. Using these, the oracle computes the correct output indexed ring element (or returns $\perp$ if the inputs are invalid). If this output already has an entry in the table $T$, then its encoding has already been determined, and can be provided as output. Otherwise, the oracle $\mathcal{R} \mathcal{G}$ chooses a new uniformly random string in $\{0,1\}^{\ell}$, outputs this string as the output encoding, and adds the appropriate entry to the table $T$.

The definition of secure obfuscation in the random GES model is as follows. 
Definition 5.2 (Virtual Black-Box in the Random GES Model). Let $\mathcal{C}=\left\{\mathcal{C}_{n}\right\}_{n \in \mathbb{N}}$ be a family of circuits and $\mathcal{O}$ a PPTM as in Definition 2.9.

A generic algorithm $\mathcal{O}^{\mathcal{R G}}$ is an obfuscator in the random generic encoding scheme model, if it satisfies the functionality and polynomial slowdown properties of Definition 2.9 with respect to $\mathcal{C}$ and to any GES oracle $\mathcal{R} \mathcal{G}$, but the virtual black-box property is replaced with:

3. Virtual Black-Box in the Random GES Model: For every (non-uniform) polynomial size generic adversary $\mathcal{A}$, there exists a (non-uniform) generic polynomial size simulator $\mathcal{S}$, such that for every $n \in \mathbb{N}$ and every $C \in \mathcal{C}_{n}$ :

$$
\left|\left(\operatorname{Pr}_{\mathcal{R G}, \mathcal{O}, \mathcal{A}}\left[\mathcal{A}^{\mathcal{R G}}\left(\mathcal{O}^{\mathcal{R G}}\left(C, 1^{n}, 1^{\lambda}\right)\right)\right]=1\right)-\left(\operatorname{Pr}_{\mathcal{R} \mathcal{G}, \mathcal{S}}\left[\mathcal{S}^{C}\left(1^{|C|}, 1^{n}, 1^{\lambda}\right)\right]=1\right)\right|=\operatorname{negl}(\lambda)
$$

We remark that while it makes sense to allow $\mathcal{S}$ to access the oracle $\mathcal{R} \mathcal{G}$, this is in fact not necessary. This is since $\mathcal{R} \mathcal{G}$ can be implemented in polynomial time (as described above), and therefore $\mathcal{S}$ can just implement it by itself.

\subsection{Security of ConjObf}

Theorem 5.3 (Security against Generic Adversaries). The algorithm ConjObf is a secure black-box obfuscator for conjunctions in the generic GES model.

Proof. Fix a security parameter $\lambda$, and the obfuscation of a conjunction $C$. The obfuscation is generated by choosing parameters for a graded encoding scheme, and then picking ring elements for all encodings. For each $i \in[n], b \in\{0,1\}$, the obfuscator picks $\rho_{i, b}, \alpha_{i, b} \in R$, and also picks $\rho_{n+1}, \alpha_{n+1} \in R$. Note that these ring elements are not all independently distributed (though each of them, on its own, is uniformly random in $R$ ). For the security proof, we treat the encoded ring elements as random variables. We use $\vec{\rho}$ to denote the vector containing $\left\{\rho_{i, b}\right\}_{i \in[n+1], b \in\{0,1\}}$, and we call these the $\rho$ random variables. We use $\vec{\alpha}$ to denote the vector containing $\left\{\alpha_{i, b}\right\}_{i \in[n+1], b \in\{0,1\}}$, and we call these the $\alpha$ random variables. (For notational convenience, we use the notation $\rho_{n+1,0}=$ $\rho_{n+1,1}=\rho_{n+1}$, and $\left.\alpha_{n+1,0}=\alpha_{n+1,1}=\alpha_{n+1}\right)$.

For a conjunction $C=(W, V)$, consider the joint distribution of the $(\vec{\rho}, \vec{\alpha})$ random variables. The elements in $\vec{\rho}$ are all independent and uniformly random ring elements. For each $i \in[n]$, the $\alpha_{i, 0}, \alpha_{i, 1}$ RVs are either equal (if $i \in W$ ) or independent (if $i \notin W$ ). Finally, the $\alpha_{n+1}$ RV is always determined by the other $\alpha$ random variables $\left(\alpha_{n+1}=\Pi_{i} \alpha_{i, V[i]}\right)$.

The Distributions $D_{o b f}(C)$ and $D_{d u m m y}$. Let $D_{o b f}(C)$ denote the obfuscator's output distribution on the conjunction $C$ :

$$
\text { (params, } \left.\left\{\left(w_{i, 0}, u_{i, 0}\right),\left(w_{i, 1}, u_{i, 1}\right)\right\}_{i \in[n]},\left(w_{n+1}, u_{n+1}\right)\right)
$$

where $\left(w_{i, b}, u_{i, b}\right)$ are the encodings of $\rho_{i, b}$ and $\left(\rho_{i, b} \cdot \alpha_{i, b}\right)$ (respectively) in $S_{\mathbf{e}_{i}}$, and $\left(w_{n+1}, u_{n+1}\right)$ are the encodings of $\rho_{n+1}$ and $\left(\rho_{n+1} \cdot \alpha_{n+1}\right)$ (respectively) in $S_{\mathbf{e}_{n+1}}$. The obfuscator's output is determined by the choice of ring elements (with joint distribution as above), together with the coins (if any) used to randomize the encodings. ${ }^{8}$

In the security proof, we also consider a "dummy obfuscation" distribution $D_{\text {dummy }}$. This dummy distribution is generated by choosing all of the encoded ring elements independently and uniformly at random, and then outputting their encodings.

\footnotetext{
${ }^{8}$ Recall that, as discussed in Remark 5.1, for security proofs in the random graded encoding scheme model, we assume that the encoding of each indexed ring element is unique.
} 
The Simulator $\mathcal{S}$. For a polynomial-size generic adversary $\mathcal{A}$ (w.l.o.g. we assume that $\mathcal{A}$ is deterministic), the simulator $\mathcal{S}$ executes $\mathcal{A}$ on the dummy distribution $D_{\text {dummy }}$ with a random graded encoding scheme (GES) oracle $\mathcal{R G}$ (where $\mathcal{R G}$ is simulated by $\mathcal{S}$ ). For a conjunction $C=(W, V)$, consider the "real" distribution $\mathcal{A}\left(D_{o b f}(C)\right)$, where the randomness is over the choice of random GES oracle and the obfuscator's random coins, and a "simulated" distribution $\mathcal{A}\left(D_{\text {dummy }}\right)$, where again the randomness is over the choice of random GES oracle and the choice of random ring elements for $D_{\text {dummy }}$. See Section 5.1 for the specification of a random GES oracle.

First, observe that in both cases, the obfuscation is identically distributed: the strings $\left\{\left(w_{i, b}, u_{i, b}\right)\right\}$ and $\left(w_{n+1}, u_{n+1}\right)$, which w.h.p. are all encodings of distinct indexed ring elements, are all independent and uniformly random (over the randomness of $\mathcal{R} \mathcal{G}$ ). We emphasize that the indexed ring elements may not be independent, but their encodings under $\mathcal{R} \mathcal{G}$ are nonetheless independent uniformly random strings. The difference between the two distributions is in $\mathcal{R} \mathcal{G}$ 's answers to the adversary's queries. For example, if the adversary computes the obfuscation's output on an input $\vec{x}$ for which $C(\vec{x})=1$, then in $D_{o b f}(C)$ this unveils a non-trivial multilinear equality between the indexed ring elements encoded in the obfuscation, whereas in $D_{d u m m y}$ the indexed ring elements are independent, and there is no such multilinear equality between them. Thus, the oracle's answers allow the adversary to distinguish the two cases.

While this specific attack clearly demonstrates that the two distributions can be distinguished, it requires "knowing" an input $\vec{x}$ for which $C(\vec{x})=1$. We show that this is (essentially) always the case: for each oracle call made by $\mathcal{A}$, either its output is identically distributed in $D_{\text {obf }}(C)$ and in $D_{\text {dummy }}$ (even conditioned on past answers and table state), or the call (implicitly) specifies a polynomial-size set $X$ of inputs, such that unless $\exists \vec{x} \in X, C(\vec{x})=1$, the call's output is uniformly random and identically distributed in $D_{o b f}(C)$ and in $D_{d u m m y}$.

The high-level strategy of $\mathcal{S}$ is as follows. It feeds $\mathcal{A}$ with an obfuscation from $D_{\text {dummy }}$. As noted above, the distributions of the obfuscation and the initial state of the table $T$ in $D_{o b f}(C)$ and $D_{\text {dummy }}$ are identical. The simulator now monitors each call made by $\mathcal{A}$ to $\mathcal{R} \mathcal{G}$. For each such call, either its output and the updated table state are identically distributed in $D_{o b f}(C)$ and in $D_{\text {dummy }}$, or the call specifies a set of inputs $X$ s.t. for some $\vec{x} \in X, C(\vec{x})=1$. Moreover, for each call, $\mathcal{S}$ can efficiently find such a set $X$, and then use its black-box access to $C$ to test, for every $\vec{x} \in X$, whether $\vec{x}$ is an accepting input and $C(\vec{x})=1$. If $\mathcal{S}$ finds an accepting input, it is essentially done - it can use its black-box access to $C$ to retrieve $W$ and $V$ (see Claim 3.3), and continue the simulation perfectly as in $D_{\text {obf }}(C)$. If there is no $\vec{x} \in X$ for which $C(\vec{x})=1$, then the output and the updated table state are identically distributed in $D_{o b f}(C)$ and in $D_{d u m m y}$, and the simulator can continue its simulation as in $D_{\text {dummy }}$. Thus, the simulator can process $\mathcal{A}$ 's calls one by one, and generate a view that is statistically close to $\mathcal{A}\left(D_{o b f}(C)\right.$.

We now elaborate on the simulator's operation, and its procedures for identifying the implicit inputs specified by $\mathcal{A}$ 's oracle calls.

Characterizing $\mathcal{A}$ 's Oracle Calls. We examine $\mathcal{A}$ 's oracle calls in sequence, and consider $\mathcal{A}$ 's view when it is run on $D_{o b f}(C)$ and on $D_{d u m m y}$. For the add, negate, mult procedures, the indexed ring element in their output is a multilinear function of the $\vec{\rho}$ and $\vec{\alpha}$ random variables. For the isZero procedure, the output is a multilinear equality test on the random variables. In fact, these are multilinear functions of $(\vec{\rho}, \vec{\alpha})$ with a very specific structure. We call these cross-linear functions, see Definition 5.8 and Claim 5.9 below. The difference between $D_{o b f}(C)$ and $D_{d u m m y}$ is that these crosslinear functions may take different values because the variables in $\vec{\alpha}$ have a different distribution 
in $D_{\text {obf }}(C)$ and $D_{\text {dummy }}$. As shown in the example above, running the obfuscated program on an input $\vec{x}$ such that $C(\vec{x})=1$ ended in an isZero call specifying a multilinear constraint that was true when the random variables are distributed as in $D_{o b f}(C)$, but false in $D_{\text {dummy }}$.

We proceed as follows. Recall that the oracle $\mathcal{R G}$ maintains a table $T$ of all indexed ring elements whose encodings have already been specified (see Section 5.1). For each element in the table $T$, we consider its representation as a (cross-linear) function of the $(\vec{\rho}, \vec{\alpha})$ random variables. After the obfuscation (real or dummy) is specified, the entries in $T$ contain the indexed elements $\rho_{i, b}$ and $\rho_{i, b} \cdot \alpha_{i, b}$. As $\mathcal{A}$ makes additional oracle calls, new entries are added to the table $T$. For these new entries, we consider the representations of their indexed ring elements as cross-linear functions of $(\vec{\rho}, \vec{\alpha})$. We focus here on calls to the add, negate, mult, encRand procedures (calls to samp and isZero are handled similarly). For each such call, the inputs should be encodings in the table $T$ : for any other string, the probability that it is a valid encoding is negligible, and the oracle $\mathcal{R G}$ just answers $\perp$ (in both the real and simulated executions). Otherwise, the inputs are in the table $T$, and the output may form a new entry in $T$. The functional representation of a table entry is defined as follows:

Definition 5.4 (Functional Representation). For each entry in $\mathcal{R} \mathcal{G}$ 's table $T$, with indexed ring element $(\mathbf{v}, \sigma) \in\{0,1\}^{\tau} \times R$ and encoding $s \in\{0,1\}^{*}$, its functional representation $f(\vec{\rho}, \vec{\alpha})$ is defined recursively:

1. Initially, the only table entries are encodings that appear in the obfuscation. I.e., the entries for the ring elements $\left\{\left(\rho_{i, b},\left(\rho_{i, b} \cdot \alpha_{i, b}\right)\right)\right\}_{i \in[n], b \in\{0,1\}}$, where the variables associated with $i$ are indexed by $\mathbf{e}_{i}$. These table entries are all distinct (excet with negligible probability), and for each table entry its functional representation is simply $f(\vec{\rho}, \vec{\alpha})=\rho_{i, b}$ or $f(\vec{\rho}, \vec{\alpha})=\left(\rho_{i, b} \cdot \alpha_{i, b}\right)$ (respectively).

2. For subsequent entries that are created on $\mathcal{A}$ 's calls to $\mathcal{R G}$, their functional representation is defined recursively. E.g. for an add call, with input encodings $s_{1}$ and $s_{2}$, if $s_{1}$ or $s_{2}$ is not in $\mathcal{R G}$ 's table $T$, then that input does not represent a valid encoding, the output is $\perp$ and no new table entry is created. If the inputs are in the table $T$, let $\left(\mathbf{v}_{1}, \sigma_{1}\right)$ and $\left(\mathbf{v}_{2}, \sigma_{2}\right)$ be the indexed ring elements that they encode. If $\mathbf{v}_{1} \neq \mathbf{v}_{2}$ then again the output is $\perp$ and no new table entry is created. The remaining case is $\mathbf{v}_{1}=\mathbf{v}_{2}=\mathbf{v}$. In this case, let $f_{1}$ and $f_{2}$ be the functional representations of the input encodings. If a new table entry is created for the output, then its representation is $\left(f_{1}+f_{2}\right)$.

The cases of samp, encRand, negate, mult calls are handled similarly.

Remark 5.5. We emphasize that, for a table entry $t$, its functional representation is completely independent of the conjunction being obfuscated. Indeed, the functional representation remains unchanged even if the $(\vec{\rho}, \vec{\alpha})$ random variables are drawn uniformly at random, as in $D_{d u m m y}$.

By definition, the functional representation indeed computes the ring element in a table entry (for any setting of $(\vec{\rho}, \vec{\alpha}))$. Moreover, for any table entry, $\mathcal{S}$ can compute a polynomial-size arithmetic circuit computing that table entry's functional representation:

Claim 5.6. For any setting of the initial random variables $(\vec{\rho}, \vec{\alpha})$, and for an entry $t$ in the table $T$ containing the indexed ring element $\left(\mathbf{v}_{t}, \sigma_{t}\right)$ and with functional functional representation $f_{t}$, it is the case that $\sigma=f_{t}(\vec{\rho}, \vec{\alpha})$. 
Claim 5.7. For each entry $t$ in $\mathcal{R} \mathcal{G}$ 's table $T$, the simulator $\mathcal{S}$ can compute a polynomial-size arithmetic circuit computing its functional representation $f_{t}(\vec{\rho}, \vec{\alpha})$.

Proof Sketch. The proof is by induction, following the recursive structure of Definition 5.4:

1. Induction basis: for the obfuscation encodings that are initially in table $T, \mathcal{S}$ can compute the functional representation $\left(\rho_{i, b}\right.$ or $\left.\rho_{i, b} \cdot \alpha_{i, b}\right)$ for each of these table entries, and a polynomial-size arithmetic circuit that computes it.

2. Induction step: for one of $\mathcal{A}$ 's calls to $\mathcal{R G}$, say an add call, as in Definition 5.4 , if $\mathcal{S}$ knows functional representations $f_{1}$ and $f_{2}$ of the input arguments (and poly-sized arithmetic circuits that compute them), then it can also compute the output's functional representation $\left(f_{1}+f_{2}\right)$ (and a poly-sized arithmetic circuit that computes it).

As noted above, the functional representations of all table entries are multilinear functions on $(\vec{\rho}, \vec{\alpha})$. Moreover, they are multilinear functions with a specific structure, which we call cross-linear functions:

Definition 5.8 (cross-linear function). A function $g$ is a cross-linear term over $(\vec{\rho}, \vec{\alpha})$ if it is of the form:

$$
g=\gamma \cdot \prod_{i \in I} \rho_{i, \vec{x}[i]} \cdot \prod_{i \in I \cap A} \alpha_{i, \vec{x}[i]}
$$

where $I, A \subseteq[n+1], \vec{x} \in\{0,1\}^{n+1}$, and $\gamma \in R$.

A function $f$ is a cross-linear function of $(\vec{\rho}, \vec{\alpha})$ if it can be expressed as a sum of cross-linear terms. I.e., it is of the form:

$$
f=\sum_{j} \gamma_{j} \cdot \prod_{i \in I_{j}} \rho_{i, \vec{x}_{j}[i]} \cdot \prod_{i \in I_{j} \cap A_{j}} \alpha_{i, \vec{x}_{j}[i]}
$$

where for each $j: I_{j}, A_{j} \subseteq[n+1], \vec{x}_{j} \in\{0,1\}^{n+1}$, and $\gamma_{j} \in R$. We assume that the expansion is compact in the sense that the tuples $\left\{\left(I_{j}, A_{j}, \vec{x}_{j}\right)\right\}_{j}$ are all distinct. We call this particular expansion the cross-linear expansion of $f$.

Claim 5.9. For any entry in the table $T$, its functional representation is a cross-linear function of $(\vec{\rho}, \vec{\alpha})$.

Proof. We show that, for every entry in the table $T$, indexed by a vector $\mathbf{v}$, its functional representation is a cross-linear function of the $\rho$ and $\alpha$ variables that are indexed by vectors $\mathbf{e}_{i}$ for which $\mathbf{v}[i]=1$. The proof is by induction over the number of calls to the oracle $\mathcal{R G}$.

- For the induction basis, after the obfuscator completes its run, the only entries in the table $T$ are for the ring elements $\left\{\left(\rho_{i, b},\left(\rho_{i, b} \cdot \alpha_{i, b}\right)\right)\right\}_{i \in[n], b \in\{0,1\}}$, where the variables associated with $i$ are indexed by $\mathbf{e}_{i}$. For each such indexed ring element, its functional representation is of the form $\rho_{i, b}$ or $\rho_{i, b} \cdot \alpha_{i, b}$, which are both crosslinear functions over the variables indexed by $\mathbf{e}_{i}$. 
- For the induction step, we first handle the case of add calls. The proofs for negate and encRand are similar. The proof for mult is slightly different, and follows below. For an add call, let $s_{1}$ and $s_{2}$ be the input encodings. As in Definition 5.4, add's output is $\perp$ unless the encodings are both in the table $T$, and contain indexed ring elements $\left(\mathbf{v}, \sigma_{1}\right)$ and $\left(\mathbf{v}, \sigma_{2}\right)$. In this case, by induction, the functional representation of both input items is a cross-linear function of the $\alpha$ and $\rho$ random variables indexed by vectors $\mathbf{e}_{i}$ for which $\mathbf{v}[i]=1$. Thus, the functional representation of the sum is also a cross-linear function of these same variables.

For a call to mult, similarly to the above, the output is $\perp$ unless the inputs are encodings of table entries $\left(\mathbf{v}_{1}, \sigma_{1}\right)$ and $\left(\mathbf{v}_{2}, \sigma_{2}\right)$, and the supports of $\mathbf{v}_{1}$ and $\mathbf{v}_{2}$ (the entries on which they are 1) are disjoint. In this case, by the induction hypothesis, the functional representations of the input arguments are cross-linear functions of disjoint sets of $\rho$ and $\alpha$ variables, and we conclude that the output's functional representation is a cross-linear function of the variables indexed by $\mathbf{e}_{i}$ for which $\mathbf{v}_{1}[i]=1$ or $\mathbf{v}_{2}[i]=1$.

We note that while it is true that $\mathcal{S}$ can compute a polynomial-size circuit computing each table entry's functional representation (Claim 5.7), it does not seem that the simulator can obtain the cross-linear expansion of these functions. Indeed, the cross-linear expansion may not have a polynomial-size description.

Classifying $\mathcal{A}$ 's Oracle Queries. From Claim 5.9 we know that the functional representations of all entries in $T$ (and in particular the representations of all inputs and outputs to oracle calls) are cross-linear functions of $(\vec{\rho}, \vec{\alpha})$. For any fixed call and table state, the outputs' functional representations in the execution of $\mathcal{A}$ on $D_{\text {obf }}(C)$ and $D_{\text {dummy }}$ are identical (see Remark 5.5). This does not, however, mean that the outputs are identically distributed: as noted above, it might be the case that when the random variables are sampled as in $D_{o b f}(C)$, the dependencies between the random variables causes the functional representation to become equivalent to that of an item that's already in the table $T$. E.g., following the example outlined above, there are two distinct ways products of the random variables that evaluate to $\Pi \rho_{i, V[i]} \cdot \alpha_{i, V[i]}$. In this case, when the second product is evaluated, the output will be the encoding of an item that's already in the table. For that same call in the simulation on $D_{\text {dummy }}$, however, the random variables are independent, and the functional representation of the new output is different from all entries in the table. In this case, the output is a fresh uniformly random encoding.

This is the main challenge for the simulator $\mathcal{S}$. For each of $\mathcal{A}$ 's calls to $\mathcal{R} \mathcal{G}, \mathcal{S}$ "wants to know" whether the output is the same as an entry that's already in the table or not. We show that for a fixed table $T$, and an oracle call made by $\mathcal{A}$, there are only three possibilities:

Possibility I: Output (Always) Identical to Another Entry. This is the simplest case. Here, in both the real and simulated executions, the output's functional representation is equivalent to some other entry in $T$. Namely, taking $f_{o}$ to be the output's functional representation, there exists some entry $t$ in $T$ with functional representation $f_{t}$, such that $f_{o} \equiv f_{t}$. In this case, for both executions, the oracle outputs item $t$ 's encoding and the table remains unchanged.

We note that $\left(f_{o}-f_{t}\right)$ is a cross-linear function of $(\vec{\rho}, \vec{\alpha})$, and that for any cross-linear function there exists a simple procedure for testing whether $\left(f_{o}-f_{t}\right) \equiv 0$. 
Claim 5.10. Let $f$ be a cross-linear function of $(\vec{\rho}, \vec{\alpha})$. There exists an efficient algorithm with black-box access to $f$, such that if $f \not \equiv 0$, then with all but negl $(\lambda)$ probability, the algorithm outputs 1. Otherwise the algorithm outputs 0 .

Proof. The algorithm picks a uniformly random assignment for $(\vec{\rho}, \vec{\alpha})$ in $R=\mathbb{Z}_{p}$, and checks whether $f$ is 0 on this assignment. If so, it outputs 0 , otherwise it outputs 0 . Since $f$ is cross-linear, its total degree is at most $2(n+1)$. By the Schwartz-Zippel Lemma, if $f \not \equiv 0$ then the algorithm will output 0 with probability at most $O(n /|R|)=\operatorname{negl}(\lambda)$.

To recognize calls of type $I$, the simulator operates as follows: for each of $\mathcal{A}$ 's oracle calls, it computes the output's functional representation and then tests whether is is equivalent to the functional representation of any table entry. If so, $\mathcal{S}$ passes the call to the oracle, returns the output to $\mathcal{A}$, and continues the simulation without changing $T$.

Possibility II: Output (Always) Different from All Entries. Here, in both the real and simulated executions, the output's functional representation is not equivalent to that of any other entry in $T$. In this case, for both distributions, the oracle outputs a fresh, uniformly random encoding for that output and updates the table to include the new item.

Below, we show a simple condition that the simulator can use efficiently identify calls of this type. Once such a call is recognized, the simulator can simply pass the call to the oracle, return the output to $\mathcal{A}$, and update its view of the table $T$ to include the new indexed item and its functional representation.

Possibility III: Output Varies. This is the trickiest case: in the real execution, the output's functional representation is equivalent to some other entry in $T$, but in the simulated execution, it is not equivalent to that entry. This is problematic case, because $\mathcal{S}$ doesn't know what the output distribution is in the real execution, and can't continue the simulation.

We show that whenever this case occurs, the adversary's call specifies an efficiently recoverable input $\vec{x} \in\{0,1\}^{n}$ such that $C(\vec{x})=1$. $\mathcal{S}$ can therefore find this $\vec{x}$ and then use its $C$-oracle to recover $C$ and complete the simulation.

Answering $\mathcal{A}$ 's Oracle Queries. For a successful simulation, $\mathcal{S}$ needs to distinguish and handle cases $I I$ and $I I I$ above. For this, we use the following definitions:

Definition 5.11 ( $\rho$-monomials, full monomials). A function $f$ is a $\rho$-monomial if it is a cross-linear term in $\vec{\rho}$ (i.e. independent of $\vec{\alpha}$ ). We say that a $\rho$-monomial is full if its total degree is exactly $n+1$ (i.e. it has maximal total degree). In this case $f=\left(\left(\prod_{i \in[n]} \rho_{i, \vec{x}[i]}\right) \cdot \rho_{n+1,0}\right)$, for some $\vec{x} \in\{0,1\}^{n}$, and we say that $f$ is the full $\rho$-monomial $\vec{x}$.

Definition 5.12 (cross-linear function with a full $\rho$-monomial). We say that a cross-linear function $f$ of $(\vec{\rho}, \vec{\alpha})$ includes a full $\rho$-monomial, if in $f$ 's cross-linear expansion, there exists a $j$ such that $\gamma_{j} \neq 0$ and $I_{j}$ is of size exactly $(n+1)$ (and so the $j$-th term in the expansion is a full $\rho$-monomial).

We show that for any cross-linear function $f$, if it includes a non-full $\rho$-monomial, then (for any conjunction $C$ ) when $(\vec{\rho}, \vec{\alpha})$ are drawn as in $D_{o b f}(C)$, with all but negligible probability $f(\vec{\rho}, \vec{\alpha}) \neq 0$. Moreover, if $f$ does include a full $\rho$-monomial $\vec{x}$, then for any conjunction $C$, if $C(\vec{x})=0$, then when $(\vec{\rho}, \vec{\alpha})$ are drawn as in $D_{\text {obf }}(C)$, with all but negligible probability $f(\vec{\rho}, \vec{\alpha}) \neq 0$. This is shown 
in Claims 5.13 and 5.14. Finally, we show that there exists an efficient procedure for identifying a $\rho$-monomial of a function $f$. This is in Claim 5.15.

$\mathcal{S}$ 's strategy for simulating $\mathcal{A}$ 's oracle calls that are not of type $I$ follows from these claims. For each such call, whose output has functional representation $f_{o}$, for each each entry $t$ in the table $T$ with functional representation $f_{t}, \mathcal{S}$ can identify a $\rho$-monomial of $\left(f_{o}-f_{t}\right)$ : if it finds a non-full monomial, then by Claim 5.13, w.h.p. in both $D_{o b f}(C)$ and $D_{d u m m y}$, the indexed ring element in the call's output is not equal to the element in entry $t$ of the table. Otherwise, if $\mathcal{S}$ finds a full $\rho$ monomial $\vec{x}_{t}$ of $\left(f_{o}-f_{t}\right)$, then it uses its black-box access to $C$ to test whether $C\left(\vec{x}_{t}\right)=1$. If so, $\mathcal{S}$ can further use its black box access to extract the representation $(W, V)$ of $C$ and complete the simulation perfectly. If not, then by Claim 5.14, w.h.p. in both $D_{o b f}(C)$ and $D_{\text {dummy }}$, the indexed ring element in the call's output is not equal to the element in entry $t$. Finally, if after testing all entries in the table, $\mathcal{S}$ has not found an accepting input, then the call is of type $I I$ and the simulation can continue.

The claims follow:

Claim 5.13. Let $f$ be a cross-linear function of $(\vec{\rho}, \vec{\alpha})$ s.t. $f \not \equiv 0$. If $f$ includes a non-full $\rho$ monomial, then for any conjunction $C$, for an assignment to $(\vec{\rho}, \vec{\alpha})$ drawn as in $D_{\text {obf }}(C)$, it holds that $\operatorname{Pr}_{(\vec{\rho}, \vec{\alpha})}[f(\vec{\rho}, \vec{\alpha})=0]=\operatorname{negl}(\lambda)$.

Proof. Let $g(\vec{\rho})=\Pi_{i \in S} \rho_{i, \vec{x}[i]}$ be a non-full monomial, with $I \subsetneq[n+1]$ and $\vec{x} \in\{0,1\}^{n+1}$. Then, by definition, the cross-linear expansion of $f$ can be written as a sum:

$$
f(\vec{\alpha}, \vec{\rho})=(\gamma \cdot g(\vec{\rho}) \cdot h(\vec{\alpha}))+\sum_{j:\left(\left(I_{j} \neq I\right) \vee\left(\vec{x}_{j} \neq \vec{x}\right)\right)} \gamma_{j} \cdot \prod_{i \in I_{j}} \rho_{i, \vec{x}_{j}[i]} \cdot h_{j}(\vec{\alpha})
$$

where $\gamma \neq 0$, and where $h(\vec{\alpha}) \not \equiv 0$ can be expanded as:

$$
h(\vec{\alpha})=\sum_{k} \delta_{k} \cdot \prod_{i \in I \cap A_{k}} \alpha_{i, \vec{x}[i]}
$$

and each $A_{k}$ is a (distinct) subset of $I$.

Now consider $h(\vec{\alpha})$, where the $\vec{\alpha}$ random variables are chosen as in $D_{o b f}(C)$, for $C=(W, V)$. This means that the $\vec{\alpha}$ random variables are not independent, but rather for some $i$ 's it may be that $\alpha_{i, 0}=\alpha_{i, 1}$, and $\alpha_{n+1}=\prod_{i} \alpha_{i, V[i]}$. Other than these restrictions the random variables in $\vec{\alpha}$ are independent and uniformly random. In particular, this means that the random variables on which $h$ depends, namely $\left\{\alpha_{i, \vec{x}[i]}\right\}_{i \in I \subsetneq[n+1]}$, are all independent and uniformly random (or rather within statistical distance $\operatorname{negl}(\lambda)$ of independent and uniformly random). ${ }^{9}$ Thus $h$ is a non-zero multilinear function of indpendent uniformly random variables, and by the Schwartz-Zippel Lemma with all but $n \operatorname{egl}(\lambda)$ probability over the choice of $\vec{\alpha}$ by $D_{o b f}(C)$, we have that $h(\vec{\alpha}) \neq 0$.

Drawing $\vec{\alpha}$ as in $D_{o b f}(C)$, and setting those variables in $f$, we examine the expansion of $f$. We get that:

$$
f_{\vec{\alpha}}(\vec{\rho})=\left(\gamma^{\prime} \cdot g(\vec{\rho})\right)+\sum_{j:\left(\left(I_{j} \neq I\right) \vee\left(\vec{x}_{j} \neq \vec{x}\right)\right)} \delta_{j}^{\prime} \cdot \prod_{i \in I_{j}} \rho_{i, \vec{x}_{j}[i]}
$$

where with all but negl $(\lambda)$ probability over $\vec{\alpha}$, we have that $\gamma^{\prime} \neq 0$. In this case, since the $\vec{\rho}$ random variables are all independent and uniformly random, the $g(\vec{\rho})$ monomial in the expansion cannot be cancelled out and $f_{\vec{\alpha}}(\vec{\rho}) \not \equiv 0$. Thus when we pick $\vec{\rho}$ uniformly at random (as in $D_{o b f}(C)$ ), with all but $n e g l(\lambda)$ probability we have $0 \neq f_{\vec{\alpha}}(\vec{\rho})=f(\vec{\rho}, \vec{\alpha})$.

\footnotetext{
${ }^{9}$ The only dependence is that if for $i \in[n]$ any of the $\vec{\alpha}_{i, \vec{x}[i]}$ variables are 0 , then $\alpha_{n+1}$ is also 0 .
} 
Claim 5.14. Let $f$ be a cross-linear function of $(\vec{\rho}, \vec{\alpha})$ s.t. $f \not \equiv 0$. Suppose $f$ includes a full $\rho$-monomial $\vec{x} \in\{0,1\}^{n}$. For any conjunction $C$, if $C(\vec{x})=0$, then for an assignment on $(\vec{\rho}, \vec{\alpha})$ drawn as in $D_{\text {obf }}(C), \operatorname{Pr}_{(\vec{\rho}, \vec{\alpha})}[f(\vec{\rho}, \vec{\alpha})=0]=\operatorname{negl}(\lambda)$.

Proof. The proof is similar to that of Claim 5.13. Let $g(\vec{\rho})=\Pi_{i \in[n+1]} \rho_{i, \vec{x}[i]}$ be the full $\rho$-monomial (we take $\vec{x}[n+1]=0$ for notational convenience). Then, by definition, the cross-linear expansion of $f$ can be written as a sum:

$$
f(\vec{\alpha}, \vec{\rho})=(\gamma \cdot g(\vec{\rho}) \cdot h(\vec{\alpha}))+\sum_{j:\left(\left(I_{j} \neq[n+1]\right) \vee\left(\vec{x}_{j} \neq \vec{x}\right)\right)} \gamma_{j} \cdot \prod_{i \in I_{j}} \rho_{i, \vec{x}_{j}[i]} \cdot h_{j}(\vec{\alpha})
$$

where $\gamma \neq 0$ and $h(\vec{\alpha}) \not \equiv 0$ can be expanded as:

$$
h(\vec{\alpha})=\sum_{k} \gamma_{k} \cdot \prod_{i \in A_{k}} \alpha_{i, \vec{x}[i]}
$$

where each $A_{k}$ is a (distinct) subset of $[n+1]$.

As in Claim 5.13, if $C(\vec{x})=0$, then the input variables to $h$ are (close to) independent and uniformly random even under $D_{o b f}(C)$. Thus w.h.p. over the choice of $\vec{\alpha}$ as in $D_{o b f}(C)$, we have $h(\vec{\alpha}) \neq 0$. Fixing any such $\alpha$, we get that $f_{\vec{\alpha}}(\vec{\rho}) \not \equiv 0$, and so w.h.p. over the choice of $\vec{\rho}$ we get: $0 \neq f_{\vec{\alpha}}(\vec{\rho})=f(\vec{\rho}, \vec{\alpha})$.

Claim 5.15. Let $f$ be a cross-linear function on $(\vec{\rho}, \vec{\alpha})$. There is an efficient algorithm with blackbox access access to $f$, such that if $f$ is not a constant function, then with all but negl $(\lambda)$ probability, the algorithm outputs a $\rho$-monomial of $f$.

Proof. The algorithm proceeds as follows. Initially set $f_{0}=f, I=\Phi$, and $i=0$. Proceed in iterations $i \leftarrow 0, \ldots, n$ :

In the $i$-th iteration, set both $\rho_{i, 0}$ and $\rho_{i, 1}$ to 0 in $f_{i}$, and check whether the resulting function is identically 0 (using the algorithm of Claim 5.10):

- If not, then there must exist a $\rho$-monomial in $f_{i}$ that includes neither $\rho_{i, 0}$ nor $\rho_{i, 1}$. The algorithm sets $\vec{x}[i]=0$, sets $f_{i+1}$ to be equal to $f_{i}$, but with the variables $\rho_{i, 0}, \rho_{i, 1}$ set to 0 , and proceeds to iteration $(i+1)$.

- Otherwise (the resulting function above was not identically equal to 0 ), then there must exist a $\rho$-monomial in $f_{i}$ that includes either $\rho_{i, 0}$ or $\rho_{i, 1}$. Similarly to the above, the algorithm can determine whether there exists $\rho$-monomial that includes $\rho_{i, 0}$ (by setting $\rho_{i, 1}$ to 0 and checking that the resulting function is not always 0). If such a monomial exists, then the algorithm adds $i$ to the set $I$, sets $\vec{x}[i]=0$, sets $f_{i+1}$ to be equal to $f_{i}$, but setting $\rho_{i, 0}=1$ and $\rho_{i, 1}=0$, and proceeds to iteration $(i+1)$.

- Finally, in the remaining case, there exists a monomial that includes $\rho_{i, 1}$, the algorithm adds $i$ to the set $I$, sets $\vec{x}[i]=1$, sets $f_{i+1}$ to be equal to $f_{i}$, but setting $\rho_{i, 0}=0$ and $\rho_{i, 1}=1$, and proceeds to the next iteration.

The algorithm's output is the $\rho$-monomial $\prod_{i \in I} \rho_{i, \vec{x}[i]}$, which (by construction) is a $\rho$-monomial of $f$. Observe that unless $f$ is a constant function, with all but negligible probability the algorithm identifies a $\rho$-monomial. 


\section{Acknowledgments}

We thank the reviewers of CRYPTO 2013 for their comments. We thank Ryo Nishimaki for pointing us to the zeroing attack on [GGH13], and the authors of [CLT13] for discussing the applicability of such attack on their scheme.

\section{References}

[AW07] Ben Adida and Douglas Wikström. How to shuffle in public. In Salil P. Vadhan, editor, TCC, volume 4392 of Lecture Notes in Computer Science, pages 555-574. Springer, 2007.

[BC10] Nir Bitansky and Ran Canetti. On strong simulation and composable point obfuscation. In CRYPTO, pages 520-537, 2010.

[BFO08] Alexandra Boldyreva, Serge Fehr, and Adam O'Neill. On notions of security for deterministic encryption, and efficient constructions without random oracles. In David Wagner, editor, CRYPTO, volume 5157 of Lecture Notes in Computer Science, pages 335-359. Springer, 2008.

[BGI $\left.{ }^{+} 12\right]$ Boaz Barak, Oded Goldreich, Russell Impagliazzo, Steven Rudich, Amit Sahai, Salil P. Vadhan, and Ke Yang. On the (im)possibility of obfuscating programs. J. ACM, 59(2):6, 2012. Preliminary version in CRYPTO 2001.

[BS02] Dan Boneh and Alice Silverberg. Applications of multilinear forms to cryptography. IACR Cryptology ePrint Archive, 2002:80, 2002.

[Can97] Ran Canetti. Towards realizing random oracles: Hash functions that hide all partial information. In CRYPTO, pages 455-469, 1997.

[CD08] Ran Canetti and Ronny Ramzi Dakdouk. Obfuscating point functions with multibit output. In EUROCRYPT, pages 489-508, 2008.

[CLT13] Jean-Sebastien Coron, Tancrede Lepoint, and Mehdi Tibouchi. Practical multilinear maps over the integers. Cryptology ePrint Archive, Report 2013/183, 2013. To appear in CRYPTO 2013.

[CMR98] Ran Canetti, Daniele Micciancio, and Omer Reingold. Perfectly one-way probabilistic hash functions (preliminary version). In STOC, pages 131-140, 1998.

[CRV10] Ran Canetti, Guy N. Rothblum, and Mayank Varia. Obfuscation of hyperplane membership. In $T C C$, pages 72-89, 2010.

[DORS08] Yevgeniy Dodis, Rafail Ostrovsky, Leonid Reyzin, and Adam Smith. Fuzzy extractors: How to generate strong keys from biometrics and other noisy data. SIAM J. Comput., 38(1):97-139, 2008. Preliminary version in Eurocrypt 2004.

[DS05a] Yevgeniy Dodis and Adam Smith. Correcting errors without leaking partial information. In Harold N. Gabow and Ronald Fagin, editors, STOC, pages 654-663. ACM, 2005. 
[DS05b] Yevgeniy Dodis and Adam Smith. Correcting errors without leaking partial information. In Harold N. Gabow and Ronald Fagin, editors, STOC, pages 654-663. ACM, 2005.

[GGH13] Sanjam Garg, Craig Gentry, and Shai Halevi. Candidate multilinear maps from ideal lattices. In Thomas Johansson and Phong Q. Nguyen, editors, EUROCRYPT, volume 7881 of Lecture Notes in Computer Science, pages 1-17. Springer, 2013. See also Cryptology ePrint Archive, Report 2012/610.

[GK05] Shafi Goldwasser and Yael Tauman Kalai. On the impossibility of obfuscation with auxiliary input. In FOCS, pages 553-562, 2005.

[GR07] Shafi Goldwasser and Guy N. Rothblum. On best-possible obfuscation. In Salil P. Vadhan, editor, TCC, volume 4392 of Lecture Notes in Computer Science, pages 194213. Springer, 2007.

[HMLS10] Dennis Hofheinz, John Malone-Lee, and Martijn Stam. Obfuscation for cryptographic purposes. J. Cryptology, 23(1):121-168, 2010.

[HRSV11] Susan Hohenberger, Guy N. Rothblum, Abhi Shelat, and Vinod Vaikuntanathan. Securely obfuscating re-encryption. J. Cryptology, 24(4):694-719, 2011.

[KV94] Michael J. Kearns and Umesh V. Vazirani. An introduction to computational learning theory. MIT Press, Cambridge, MA, USA, 1994.

[LPS04] Ben Lynn, Manoj Prabhakaran, and Amit Sahai. Positive results and techniques for obfuscation. In EUROCRYPT, pages 20-39, 2004.

[Mau05] Ueli M. Maurer. Abstract models of computation in cryptography. In IMA Int. Conf., pages $1-12,2005$.

[Rot13] Ron Rothblum. On the circular security of bit-encryption. In TCC, pages 579-598, 2013.

[RW04] R. Renner and S. Wolf. Smooth renyi entropy and applications. In IEEE International Symposium on Information Theory, IEEE International Symposium on Information Theory, page 233, 2004.

[Sho97] Victor Shoup. Lower bounds for discrete logarithms and related problems. In EUROCRYPT, pages 256-266, 1997.

[Wee05] Hoeteck Wee. On obfuscating point functions. In Harold N. Gabow and Ronald Fagin, editors, STOC, pages 523-532. ACM, 2005. 